\title{
Stochastic epidemic metapopulation models on networks: SIS dynamics and control strategies
}

\author{
Andrew L. Krause, Lawrence Kurowski, Kamran Yawar, and Robert A. Van Gorder* \\ Mathematical Institute, University of Oxford, Andrew Wiles Building, Radcliffe Observatory Quarter, Woodstock Road, Oxford, OX2 6GG, UK \\ *Robert.VanGorder@maths.ox.ac.uk
}

\begin{abstract}
While deterministic metapopulation models for the spread of epidemics between populations have been well-studied in the literature, variability in disease transmission rates and interaction rates between individual agents or populations suggests the need to consider stochastic fluctuations in model parameters in order to more fully represent realistic epidemics. In the present paper, we have extended a stochastic SIS epidemic model - which introduces stochastic perturbations in the form of white noise to the force of infection (the rate of disease transmission from classes of infected to susceptible populations) - to spatial networks, thereby obtaining a stochastic epidemic metapopulation model. We solved the stochastic model numerically and found that white noise terms do not drastically change the overall long-term dynamics of the system (for sufficiently small variance of the noise) relative to the dynamics of a corresponding deterministic system. The primary difference between the stochastic and deterministic metapopulation models is that for large time, solutions tend to quasi-stationary distributions in the stochastic setting, rather than to constant steady states in the deterministic setting. We then considered different approaches to controlling the spread of a stochastic SIS epidemic over spatial networks, comparing results for a spectrum of controls utilizing local to global information about the state of the epidemic. Variation in white noise was shown to be able to counteract the treatment rate (treated curing rate) of the epidemic, requiring greater treatment rates on the part of the control and suggesting that in real-life epidemics one should be mindful of such random variations in order for a treatment to be effective. Additionally, we point out some problems using white noise perturbations as a model, but show that a truncated noise process gives qualitatively comparable behaviors without these issues.
\end{abstract}

Keywords: stochastic epidemic model; susceptibles-infectives-susceptibles (SIS) dynamics; epidemics on networks; control of epidemics

\section{Introduction}

The most fundamental epidemic models, first presented by Kermack and McKendrick (1927), are still used as a basis for the majority of new epidemic models proposed today (Brauer, 2017). In these models the populations are divided into different compartments; hence they are generally referred to as compartmental models. Traditional compartmental models assumed 'mixed populations' (Newman, 2002), meaning that each individual in the population had the same probability to catch the infection or transfer the infection onward to a susceptible individual. Such models do not account for spatially structured populations, or for variations in the transmission rates between individuals. The SIS model is one widely used example, and has been applied to diverse areas from the spread of sexually-transmitted diseases (Hethcote and Yorke, 1984) to internet viruses (Berger et al., 2005).

In order to describe epidemiological phenomena, it is useful to consider the SIS model on networks. A common approach is to describe such a model with each node in the network representing one individual who can be either infected or not; this model has been widely discussed (Keeling and Eames, 2005); this approach is in particular useful when investigating virus spread across the internet via emails (Berger et al., 2005). An alternative is the so-called meta-population approach, where we consider spatially structured interacting subpopulations, such as cities, urban areas, geographical locations with high population density, and so on (Ball et al., 2015; Grenfell and Harwood, 1997; Pastor-Satorras et al., 2015). We note that these approaches make use of deterministic and stochastic processes with an underlying network topology representing the interactions between populations (Keeling et al., 2004). Stochastic metapopulation models (Ball et al., 2004, 2010), and deterministic metapopulation models (Pastor-Satorras et al., 2015; Sanders et al., 2012), both consider multiple interacting populations with infection occurring within and between nodes. In (Arrigoni and Pugliese, 2002), the authors consider various asymptotic limits of a stochastic metapopulation model, and in some cases arrive at a deterministic model such as the those mentioned.

We can introduce stochastic terms to the SIS model to reflect the randomness in the number of encounters between healthy and sick individuals in a population. Two common mathematical approaches to analyzing such stochastic SIS systems are often proposed. The first approach is to model the disease spread as a Markov jump process (Allen and Burgin, 2000). For a large population, there are multiple infections possible in 
a short time, whence we need to consider a large event space and hence the Markov chain approach becomes computationally intractable. Khanafer and Basar (2014) describe an SIS model on a network where each node represents an individual agent. They view each node as a Markov chain with two states: infected and cured. They also assume the infection and recovery rates are Poisson processes. The second approach makes use of stochastic differential equations (Gray et al., 2011) to model the entire population, rather than individual agents. One assumes that the population is large enough for it to make sense to consider the densities of the sick and healthy populations, rather than individual agents (Keeling and Ross, 2008). We note that similar population-level stochastic noise was used in several studies to represent uncertainty in model parameters (Yang and Mao, 2013; Chen and Li, 2009).

There are various methods of curbing disease spread via implementing control policies, such as slaughtering infected animals and restricting herd movement between farms as outlined by Ferguson et al. (2005) for the case of foot-and-mouth disease in a population of pigs. Controlling disease spread on a network can be done by introducing an antidote or vaccine at certain nodes (Borgs et al., 2010). It is desirable, both to reduce economic costs and to minimize the use of environmentally detrimental pesticides, to seek optimal control policies which will minimize the time to alleviating or eliminating the infection at minimal cost (Forster and Gilligan, 2007b). It is also possible to control the epidemic by modifying the network configuration itself (Sanders et al., 2012).

Control of SIS contagions on networks was discussed by Nowzari et al. (2016). While optimal control of contagions have been considered under the standard SIS model (Forster and Gilligan, 2007a; Kim et al., 2006; Nowzari et al., 2016) corresponding to SIS dynamics at a single node, there remains much work to be done when attempting to control the spread of SIS contagions on networks (Pellis et al., 2015). SIS dynamics on network domains was considered by Khanafer and Basar (2014), who use an SIS n-intertwined Markov model (Van Mieghem et al., 2009; Van Mieghem, 2011) to replicate the spread of infection. In this model each node on the network can be viewed as a Markov chain with an infected and cured state that has two different Poisson processes which are responsible for driving the curing and infection rates (Khanafer and Basar, 2014). Eshghi et al. (2015) consider a similar problem, but in the context of malware spread in computer networks. Shaw and Schwartz (2010) consider a modification of SIS and SIRS models created by adding a vaccinated class, before implementing a control through vaccination. They make use of adaptive networks where the non-infected nodes rewire in an adaptive manner to reduce connection with the infected nodes, and find that adaptive networks along with random immunization is much more effective compared to static networks with vaccination. SIRS epidemic models with the incorporation of vaccination have also been studied on complex networks (Chen and Sun, 2014a,b). Tomovski and Kocarev (2012) designed an algorithm for virus control on complex networks.

Stochastic structured population models exist in the literature, including models with household structure (Ball et al.,
2004, 2010). We emphasize that this kind of forcing is demographic stochasticity, where the random forcing is due to individual interactions, and so these are typically accounted for in a discrete way (Keeling et al., 2004). To our knowledge, a stochastic metapopulation SIS model with parameter uncertainty has not been discussed in the literature. In this paper we shall extend the stochastic SIS epidemic model of Gray et al. (2011) to spatial networks, thereby obtaining a stochastic epidemic metapopulation model. In Section 2 we review useful background notation and knowledge of the dynamics of deterministic metapopulation SIS models on networks. Then, we define the stochastic SIS model on networks in Section 3, where we also numerically simulate the dynamics emergent from such a model and compare these dynamics to the deterministic model. In Section 4 we introduce two families of controls, namely bang-bang controls (where either all or none of the treatment is provided) and feedback controls (where the rate of treatment is permitted to vary based on the present state of the epidemic). Either is used in conjunction with a variety of information structures which trigger the treatment. In Section 5 , we outline some problems with the typical approach used in these kinds of stochastic perturbation models, and develop a truncated noise model that alleviates these concerns while giving essentially identical model outputs. We discuss the results and give concluding remarks in Section 6.

\section{Metapopulation SIS Models}

In this section we review useful results from the literature on SIS epidemic models, and also define the notation which shall be essential for understanding the stochastic model of the next section.

\subsection{Compartmental models}

A common and simple way to describe disease spread in a population of a communicable disease is by using compartmental models, where we divide a population into compartments, or classes. In the SIS model the population is divided into two sets: infectives (sick) and susceptibles (healthy) (Allen and Burgin, 2000). We assume infection does not confer immunity; that is, that the passage of individuals from the susceptible class $S$ to the infected class $I$ and then back to class $S$ is possible. SIR (susceptible-infected-removed) is a similar, three-compartment model, where individuals removed from class $I$ are placed in the removed class $R$, either immune to the disease or dead. More compartmental models have been considered, such as SEIR where it takes time for individuals in the exposed class $E$ to catch the catch the disease and be placed in class $I$, among other variations (Martcheva, 2015). Here we will restrict to the SIS class of models as it provides a simple demonstration of the coupled effects of spatial networks and stochasticity that we are interested in. Similarly, we will ignore issues of demographics such as births, deaths, and heterogeneity within the population. 


\subsection{SIS dynamics on a single node}

We first consider a single isolated node, in order to define relevant notations. Consider a population of $N(t)$ individuals partitioned into two compartments of susceptibles $S(t)$ and infected individuals $I(t)$. Let us assume there are no births and deaths, so the total population size $N(t)=I(t)+S(t)$ remains constant in time. Let $\hat{\beta} \geq 0$ be the force of infection, which is the rate of disease transmission between individuals from classes $S$ and $I$ and is measured in infections per individual per second. Assume $\hat{\delta} \geq 0$ is the recovery rate measured in $s^{-1}$. Finally, let $\hat{t} \geq 0$ be the time in $s$. The differential equations describing this system are (Gray et al., 2011)

$$
\frac{d S(\hat{t})}{d \hat{t}}=\hat{\delta} I(\hat{t})-\hat{\beta} S(t) I(t) \text { and } \frac{d I(t)}{d \hat{t}}=-\hat{\delta} I(\hat{t})+\hat{\beta} S(\hat{t}) I(\hat{t})
$$

where $S(0)=S_{0}, I(0)=I_{0}$. Since $N=S(t)+I(t)$ for all $t$ and in particular $S_{0}=N-I_{0}$, equation (1) can be reduced to

$$
\frac{d I(t)}{d \hat{t}}=\hat{\beta}(N-I(\hat{t})) I(\hat{t})-\hat{\delta} I(\hat{t})
$$

with $I(0)=I_{0}$. We can nondimensionalize and consider only population densities by setting

$$
t=\frac{\hat{t}}{T}, p=\frac{I}{N}, \delta=\hat{\delta} T, \beta=\hat{\beta} N T, p_{0}=\frac{I_{0}}{N},
$$

where $T$ is an arbitrary time scale so that the infection and recovery rates are nondimensional. We then have

$$
\frac{d p}{d t}=\beta p(1-p)-\delta p
$$

and $p(0)=p_{0}$, where $p \in[0,1]$. If the infection rate is larger than the recovery rate $(\beta>\delta)$, the solution is a logistic growth curve, with the infection persisting in the long run. If the opposite is true $(\delta>\beta)$, the infected population decays exponentially to zero.

\subsection{Deterministic metapopulation SIS model on networks}

Networks have been increasingly used to describe a variety of phenomena by elucidating the role that structure plays in many physical and virtual systems (Newman, 2010). We can also use networks to describe networks of cities or areas of high population density, connected together by transport links such as roads or airplane routes, represented by edges of the network. This is the so-called "metapopulation" model where we will assume there is some fixed population at each node (Pastor-Satorras et al., 2015). More abstractly, nodes can also represent communities with edges between them modeling interactions (Lajmanovich and Yorke, 1976), or even hosts within which pathogen populations are accounted for (McCormack and Allen, 2006). While such models are becoming more popular, there are still many important questions about incorporating different effects (e.g. stochasticity, nonlinearity) within the setting of a structured population (Ball et al., 2015).
For a network of $K$ nodes it is common practice to describe a network by a square adjacency matrix $A=\left(a_{i, j}\right)_{i, j=1, \ldots, K}$, defined to be such that

$$
a_{i, j}=\left\{\begin{array}{l}
1, \text { if there is an edge linking nodes } i, j, \\
0, \text { otherwise. }
\end{array}\right.
$$

In this paper we will focus on undirected graphs, that is, on networks where infection can be transmitted along all edges in either direction; so if nodes $i$ and $j$ are connected with edge $(i, j)$, so are nodes $j$ and $i$; this implies that the adjacency matrix describing the network is symmetric. In addition, we will assume that all nodes are connected to themselves, so $a_{i, i}=1$ for all $i$. This implies disease can be transmitted within a node from infected individuals already present there.

Consider a network of $K$ nodes, described by a symmetric adjacency matrix $A$. Let $p_{k}$ be the density of the infected population at node $k$, and $\mathcal{I}_{k}$ be the index set of nodes connected with node $k$, where $1 \leq k \leq K$. We will assume that $k \in I_{k}$ for all $k$. We will assume that at node $k$, infections happen due to mixing of individuals at node $k$ and at all nodes connected to $k$ ("neighbors" of node $k$ and node $k$ itself), that is at all nodes $j$ such that $j \in \mathcal{I}_{k}$. Let $\beta_{k j}$ be the rate of infection along edge $(j, k)$ and $\delta_{k}$ the recovery rate at node $k$. We therefore extend equation (4) for the dynamics at node $k$ as follows:

$$
\frac{d p_{k}(t)}{d t}=\left(1-p_{k}(t)\right) \sum_{j \in \mathcal{I}_{k}} \beta_{k j} p_{j}(t)-\delta_{k} p_{k}(t),
$$

for $k=1, \ldots, K$. We remark that the use of an adjacency matrix $a_{k j}$ or the adjacent neighbors of a node $\mathcal{I}_{k}$ is redundant given $\beta_{k j}$, as non-adjacent nodes can simply be assumed to have $\beta_{k j}=$ 0 .

The metapopulation model given by (6) is suitable for several biological interpretations, such as geographical connectivity between cities, households, or pathogen populations within individual hosts (Ball et al., 2015). It is not clear a priori how to determine the infection rates, $\beta_{k j}$, and assigning particular values to these rates corresponds not just to a biological interpretation of the metapopulation structure, but also to additional assumptions about within-population contact rates and population mixing. Even restricting to the spatial interpretation of the metapopulation model, this is still an open problem (Keeling and Rohani, 2002; Riley et al., 2015). There have been some attempts to relate commuter and individual movement to determining contact rates between nodes (Keeling et al., 2010).

For concreteness, we will analyze a particular model for $\beta_{k j}$ corresponding to homogeneous populations with identical contact rates between individuals, which we will denote by $\beta$ (note that we have assumed each node to have the same population, and nondimensionalized by this in writing $\beta$ as a scalar). In this case we have,

$$
\beta_{k j}= \begin{cases}\frac{\beta}{d_{k}}+\frac{\beta}{d_{j}}, & k \neq j, \\ \frac{\beta}{d_{k}}, & k=j,\end{cases}
$$

where $d_{k}$ and $d_{j}$ denote the degrees of nodes $k$ and $j$ respectively. We note that the calculation of the degree of each node 
includes self-edges, and these arise to account for correct proportional interactions between populations due to contacts between nodes. We emphasize that in general these rates will be heterogeneous due to heterogeneity in populations or in contact rates between nodes due to, e.g., environmental factors. In general one could consider time-varying infection rates to account for seasonality, day/night cycles, and other topological effects in a dynamic network setting (Kiss et al., 2012; Pastor-Satorras et al., 2015), but we do not pursue this here.

There are many well-known stability results for deterministic SIS processes. In the scalar case of Equation (4), there exists a simple basic reproduction number, $\mathcal{R}_{0}=\beta \delta^{-1}$, which determines the long-time dynamics of the disease process. The disease-free equilibrium, $p^{*}=0$, is globally asymptotically stable if and only if $\mathcal{R}_{0} \leq 1$, and the endemic equilibrium, $p^{*}=1-\mathcal{R}_{0}^{-1}$, is globally asymptotically stable if $\mathcal{R}_{0}>1$, (as long as there is some initial infected population $p(0)>0$ ) (Brauer, 2017; Diekmann and Heesterbeek, 2000; Martcheva, 2015).

The original calculation of the basic reproduction number for an SIS process in a collection of $n$ populations was done by Lajmanovich and Yorke (1976), and we review their results here. We define a matrix corresponding to Equations (6) by $B_{k j}=\beta_{k j}-\operatorname{diag}\left(\delta_{k}\right)$. We denote the eigenvalue with largest real part by $\lambda_{1}(B)$. We then have that this eigenvalue is equivalent to the basic reproduction number, so that for $\lambda_{1}(B) \leq 0$, the disease-free equilibrium, $p_{k}^{*}=0$ is globally asymptotically stable. The endemic equilibrium cannot be explicitly computed for an arbitrary adjacency matrix $\beta_{k j}$, but a unique equilibrium exists and, this equilibrium is globally asymptotically stable if $\lambda_{1}(B)>0$. This result, as in the scalar case, gives a complete characterization of the long-time behavior of the SIS process on heterogeneous networks. Recent reformulations and proofs of these results have also been carried out by Khanafer et al. (2014) and Van Mieghem and Omic (2013).

\section{Stochastic Metapopulation SIS Models}

Until now we have assumed that there is a constant rate of encounters between infected and susceptible individuals resulting in new infections. It may be more realistic to consider the case where there is some random variation in this proportion. For the meta-population model in particular it seems reasonable to assume that the rate of cross-edge infection is random rather than fixed-for example, there is a degree of randomness to how many people from two cities meet as result of traveling. We can express this by assuming that the infection rate is in fact a stochastic variable (Dalal et al., 2007). At the same time, the recovery rate is disease-specific and thus it is natural to assume it is constant.

Allen and Burgin (2000) discuss a stochastic SIS model derived as an SDE (stochastic differential equation) approximation to a continuous-time Markov-jump process, where the infected population can jump between states according to transition probabilities. In principle, for a population of $N$ individuals, we have $2^{N}$ possible states and thus need as many transition probabilities to describe the disease evolution. This can be simplified by considering $K$ groups of individuals which are indistinguishable within a group, and only considering a probability for the number of infected individuals for each group. While the Markov-chain approach can be an intuitively simple way of introducing stochasticity in disease dynamics (for example, it can be understood as a natural extension of a random walk model), an alternative approach via parameter perturbation of a metapopulation model is numerically more efficient for large $N$. This approach is derived for a single-population model by Gray et al. (2011). We will follow the reasoning of Gray et al. (2011) to first understand their model for a single node. We will then extend it to networks of $K$ nodes.

Gray et al. (2011) introduce stochasticity to (4) via white noise terms. In deriving (4) we assumed that $\beta$ is the infection rate, so that with

$$
d p=p(1-p) \beta d t-p \delta d t
$$

$p(0)=p_{0}$ we can treat $\beta d t$ as the proportion of individuals infected in the small time $d t$. If we now perturb $\beta$ stochastically, we can instead write this proportion as $\tilde{\beta} d t$ with (Gray et al., 2011)

$$
\tilde{\beta} d t=\beta d t+\sigma d W(t),
$$

where $W(t)$ is a Brownian motion. This means that $\tilde{\beta} d t$ is normally distributed, with mean

$$
\mathrm{E}(\tilde{\beta} d t)=\mathrm{E}(\beta d t+\sigma d W(t))=\beta d t
$$

(by linearity of expectation) and variance

$$
\operatorname{Var}(\tilde{\beta} d t)=\operatorname{Var}(\beta d t+\sigma d W(t))=\sigma^{2} d t
$$

The rate of infection-resulting encounters is still centered around $\beta$, but is no longer fixed. Implementing this idea on a single node yields the SDE

$d p=p(1-p)(\beta d t+\sigma d W)-\delta p d t=p(\beta(1-p)-\delta) d t+\sigma p(1-p) d W$,

which is a stochastic differential equation for $p$. We note that equation (12) is understood with respect to Itô integration (Øksendal, 2003), and this is true for all subsequent stochastic differential equations in the present paper.

We can associate a white noise term with each edge in our network models. This accounts for both random variation in the infection-resulting encounters within each node as well as the inter-node encounters. We assign a random infection rate $\tilde{\beta}_{k j}$ to each edge $(k, j)$ of the network, defined by

$$
\tilde{\beta}_{k j} d t=\beta_{k j} d t+d W_{k j}(t)
$$

where $1 \leq k, j \leq K, \beta_{k j}$ is a positive constant and the $W_{k j}$ are independent Brownian motions. Replacing the constant infection rates $\beta_{k j}$ in equation (12) with the random infection rates $\tilde{\beta}_{k j}$ we have

$$
d p_{k}=\left(1-p_{k}\right) \sum_{j \in \mathcal{I}_{k}} p_{j}\left(\beta_{k j} d t+\sigma_{k j} d W_{k j}\right)-\delta_{k} p_{k} d t
$$


for $k=1, \ldots, K$. Equation (14) constitutes a stochastic metapopulation model model for SIS epidemics, and serves as an extension of Gray et al. (2011) to spatial network domains. As in the computation of the contact rates in the deterministic model given by (7), we assume that there is some per-capita variation in the contact rate between individuals that we denote $\sigma$. We compute the standard deviations of the white noise processes at the edges as,

$$
\sigma_{k j}= \begin{cases}\frac{\sigma}{d_{k}}+\frac{\sigma}{d_{j}}, & k \neq j, \\ \frac{\sigma}{d_{k}}, & k=j .\end{cases}
$$

Gray et al. (2011) prove several theorems related to the longtime stability of solutions to the scalar Equation (12). In particular they define a stochastic basic reproduction number which in our notation is $\mathcal{R}_{0}^{S}=\mathcal{R}_{0}-\sigma^{2}(2 \delta)^{-1}=\beta \delta^{-1}-\sigma^{2}(2 \delta)^{-1}$ such that if $\mathcal{R}_{0}^{S}<1$ and $\sigma<\beta$, then for any initial data $p(0) \in(0,1)$, the solution $p(t)$ to Equation (12) exponentially tends to zero almost surely (with probability one). They also show that the same long-time exponential extinction occurs if $\sigma^{2}>\max \left(\beta, \beta(2 \delta)^{-1}\right)$. Finally, Gray et al. (2011) prove that if $\mathcal{R}_{0}^{S}>1$, then there exists a unique stationary distribution which corresponds to a positive endemic state, and they characterize it by its mean and variance.

We remark that this change in the basic reproduction number is due to the nature of the noise process in the Itô formulation of the SDE. To see this, we follow Gray et al. (2011) and write equation (12) near the disease-free equilibrium $p=0$ as

$$
d p=p((\beta-\delta) d t+\sigma d W)
$$

which is a geometric Brownian motion. Note that (16) has the exact solution

$$
p(t)=p_{0} \exp \left(\left((\beta-\delta)-\frac{\sigma^{2}}{2}\right) t+\sigma W_{t}\right),
$$

which immediately suggests that the $\mathcal{R}_{0}^{S}$ described above correctly captures the epidemic process. This also suggests that the parameter uncertainty introduced by this noise term has to carefully be assessed in comparison with classical theory on the basic reproduction number (Heesterbeek and Dietz, 1996).

We note that the effect of the noise, as long as the variance $\sigma<\beta$, is simply to reduce the basic reproduction number. These results suggest that disease processes under environmental stochasticity will be less likely to reach an endemic state, at least in the scalar case. We conjecture that qualitatively similar results hold for our network-structured model given by Equation (14). In particular, we suspect (and numerically demonstrate evidence) that the basic reproduction number for our stochastic network model will be less than the basic reproduction number of the deterministic SIS process on a network described at the end of Section 2.3. We also will demonstrate modeling problems in using white noise processes to model parameter perturbation, and suggest a remedy in Section 5 .

In what follows, we will consider the system given by (14) on different networks of $K$ nodes. Throughout, we will assume

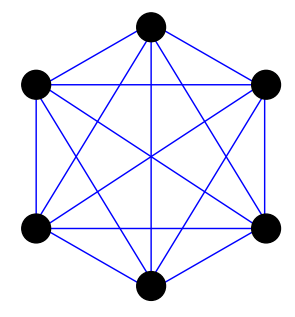

(a)

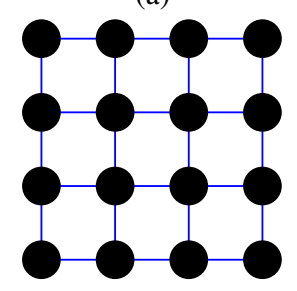

(c)

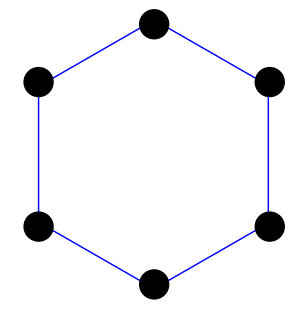

(b)

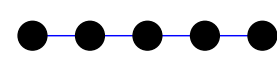

(d)
Figure 1: Examples of (a) complete, (b) cycle, (c) lattice, and (d) path graphs, which shall be the networks we consider.

that for all $k, j=1 \ldots, K, \delta_{k}=\delta$ and the infection rates between nodes are determined by (7) and (15) for scalars $\beta$ and $\sigma$ respectively. While other forms of network heterogeneity can be important in understanding epidemic processed (Lajmanovich and Yorke, 1976; Khanafer and Basar, 2014), here we are primarily interested in deviations between network topologies for a given homogeneous group of subpopulations. We will always assume that $p_{j}(0)=0.1, j=1, \ldots, K$. Each edge will have a different Brownian path driving the stochastic infection between nodes, and each edge will have a mean infection rate given by (7) with variance given by (15).

\subsection{Numerical approach}

We solve Equations (14) using the Euler-Maruyama scheme (Higham, 2001), with a step-size of $\Delta t=10^{-4}$. For specific simulations we also checked pathwise convergence for decreasing values of $\Delta t$ (Grüne and Kloeden, 2001; Kloeden and Neuenkirch, 2007). We generate different Brownian paths for each edge in a given network using the same seed in our random number generator for varying parameters in order to understand the effects these parameters have on a particular realization. To average across different realizations we vary the underlying random number generator's seed. Finally, our deterministic results were computed using the MATLAB function ODE45 which implements a high-accuracy Runge-Kutta scheme. We compared these deterministic simulations with stochastic simulations with $\sigma=0$, and, as expected, only observed transient errors of the order of the time step $\Delta t$. In all cases tested, identical steadystate values are reported by both algorithms.

We remark that other approaches can be used to simulate our model and related model formulations. In particular, Gillespietype methods are frequently used to sample continuous-time Markov Chain formulations of epidemic models, where a timeto-event is computed based on the probability of infection (e.g. the contact rate) (Allen, 2017). Our model is concerned with a continuous state space, and so directly solving the SDEs is 
sufficient to capture the behaviors we are interested in demonstrating. Additionally, while there is a growing literature on numerical simulation of SDEs using a variety of methods, we are primarily interested in demonstrating generic model behaviors rather than computational efficiency. See Higham (2001) for some discussion and references to more computationally efficient methods to simulate SDEs.

\subsection{Large-time qualitative dynamics}

Figure 1 gives examples of the types of networks considered in our numerical simulations. A path network is the same as a cycle network with a single edge removed. In a (square) lattice network, edges along the outer boundary of the network each have three neighbors, all nodes in the middle of the network have exactly four neighbors and the four vertices have only two. Finally, in complete networks all nodes are connected with all other nodes, or equivalently the adjacency matrix $A=\left(a_{i, j}\right)$ is such that $a_{i, j}=1$ for all $i, j$.

We now numerically investigate how network structure impacts the long-term dynamics of the solutions to equation (12). We plot several time series of infected populations at each node, $p_{k}(t)$, in Figure 2(a-d). The colored solid lines correspond to stochastic simulations with $\sigma=0.02$, while the black dashed lines correspond to deterministic simulations. While there are $|K|=9$ dashed black lines in each graph, many nodes in the deterministic case will have the same or approximately the same value, and so there appear to be fewer dashed lines. The insets are over the last 10 units of time to demonstrate the long-time behavior of the model. We include nodal plots of the steady state values of $p_{k}(t)$ for the square lattice and path graphs to demonstrate these spatially heterogeneous populations (see Figure 2(e-f)). We note that the infected populations on the cycle and complete graph do not have any spatial heterogeneity in the deterministic model due to the uniform degree distribution. In all simulations the stochastic nodal populations fluctuate about the deterministic steady state, essentially inheriting any heterogeneity (or lack thereof) due to the underlying network topology and the contact rates given by (7).

Note the variation in the axes of the infected populations in the insets of Figure 2(a)-(d), which is due to different 'effective' infectivities corresponding to the network structures used. The complete and cycle graphs tend to fluctuate about a single deterministic value of $p_{k}(t)$ for all nodes after a short transient period, whereas the path and square lattice graphs have an induced heterogeneity in the nodal infected populations due to differences between the number of neighboring nodes in each case. Nodes with higher degrees typically have a larger infected population in both the deterministic and stochastic cases, but we note that because of the way contact rates are defined in (7) and (15), there is some nontrivial heterogeneity in the mean infected population. In particular, note that the nodes with the largest infected population in the path graph are those neighboring the boundary nodes, as populations there will have more frequent contact with the boundary nodes due to our assumption about homogeneous contact rates. We also note that for this value of $\sigma$, the network topology leads to a much greater difference between the infected populations at different nodes than almost all temporal differences between nodal populations due to the stochasticity.

We also observe noise-induced extinction as described in Gray et al. (2011), where the corresponding deterministic disease persists. These stochastic bifurcation phenomena have recently been explored in population ecology as a way to understand when variance in a population can lead to extinction, as opposed to changes in mean population inducing extinction (Gellner et al., 2016). We plot simulations of the same system with two different realizations on a path graph in Figure 3 with $|K|=3$. These results demonstrate that near the stability boundary in the deterministic setting, corresponding to low infection rates at each node, stochastic variation can lead to extinction or multiple recurrence of higher levels of the disease. We suspect that the theoretical results of Gray et al. (2011) can be extended to the network setting so that for large enough $\sigma$, the disease will eventually die off as $t \rightarrow \infty$. However, Figure 3b demonstrates extremely long timescales for the disease to eventually become extinct, which suggests that on physically-meaningful time scales recurrence of the disease over time can occur, as the spikes observed in the nodal infection levels correspond to large values of the disease prevalence compared to the deterministic steady state. Finally we remark that the network setting given by Equation (14) differs from the scalar case of Equation (12) as if a node is near the disease-free absorbing state, the noise term can locally appear like additive (as opposed to multiplicative) noise if a neighboring node is has a large infected population. This may lead to a resilience against noise present in the stochastic model, and it also leads to some difficulties numerically integrating it as additive noise can lead to negative values of $p_{k}(t)$ if the time step is not sufficiently small. We discuss this in more detail in Section 5.

We now consider the long-term behavior of solutions to these systems for a range of values of $\delta, \beta$ and $\sigma^{2}$. We first consider the deterministic network model given by equations (6), which we know to have globally-attracting solutions for all values of the infection rate $\beta$ and recovery rate $\delta$ (e.g. through Lyapunov analysis (Khanafer et al., 2014; Van Mieghem and Omic, 2013)), either corresponding to extinction of the disease or an endemic equilibrium. We define the nodal average infection by

$$
\bar{p}=\frac{1}{K} \sum_{j=1}^{K} p_{k}\left(t_{f}\right),
$$

where $K$ is the number of nodes in the network, and $t_{f}$ is the final simulation time, which we take to be $t_{f}=2000$, as all simulations computed are within numerical precision of their steady state value.

The value $\bar{p}$ is the long-term mean contagion across the network and we plot bifurcation diagrams in Figure 4 for the deterministic model $(\sigma=0)$ on $n=16$ and $n=25$ nodes using this mean contagion. On these plots we also provide the lines which denote the stability change between disease-free and endemic equilibria given at the end of Section 2.3, as well as a plot of the deterministic basic reproduction number $\mathcal{R}_{0}$ as a function of both network topology and the number of nodes $n$. As one would expect, this is the same for all network topologies for 


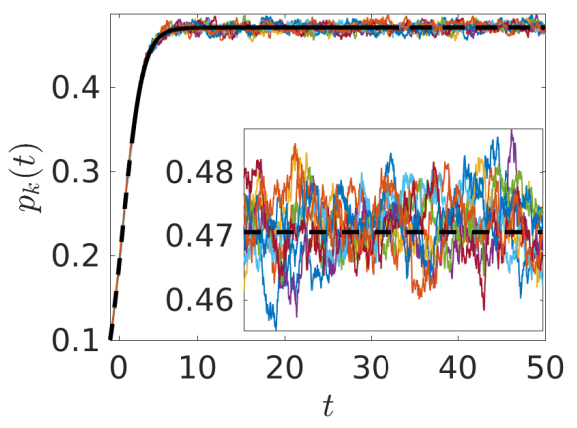

(a) Complete Graph

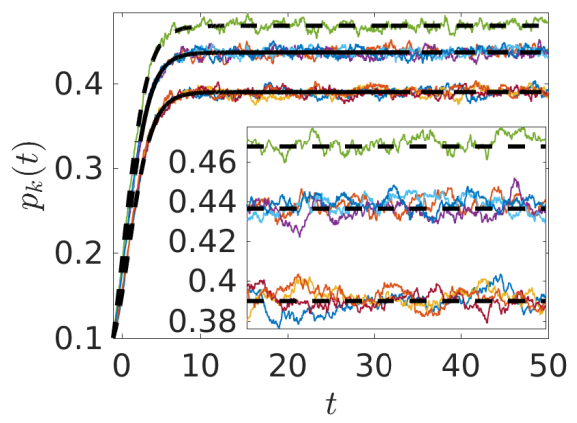

(c) Square Lattice Graph

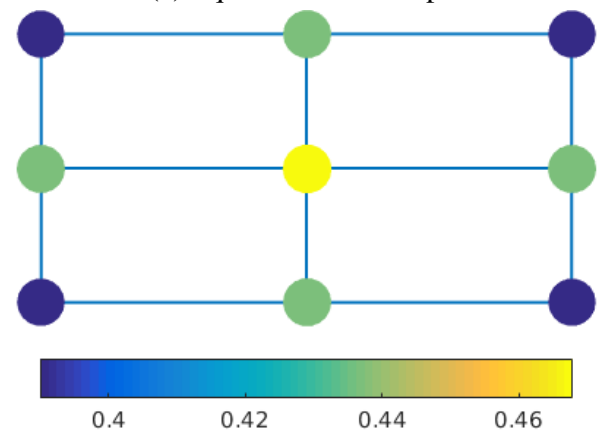

(e) Square Lattice Graph

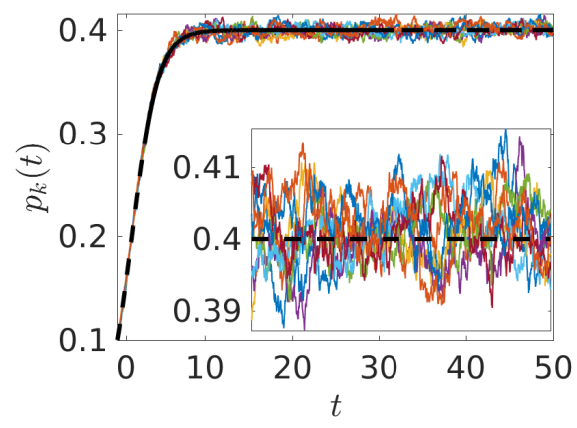

(b) Cycle Graph

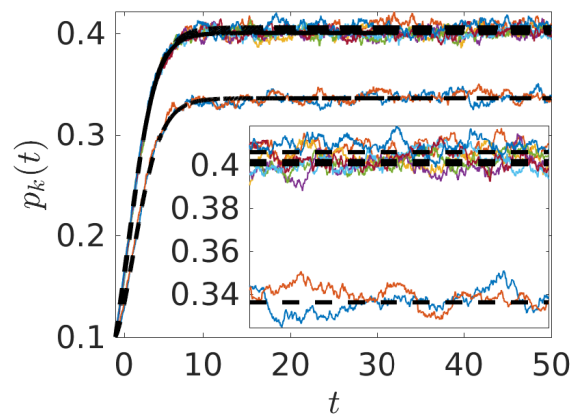

(d) Path Graph

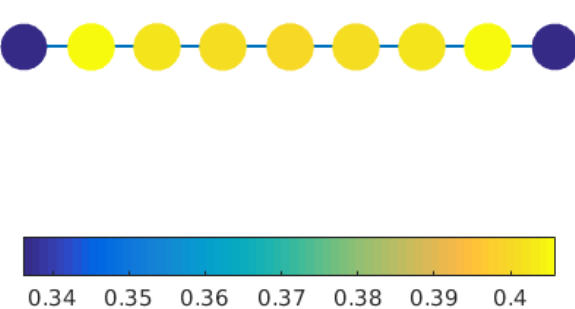

(f) Path Graph

Figure 2: Time series of nodal values of the infected populations $p_{k}(t)$ in four different graphs. Solid lines correspond to stochastic simulations using the EulerMaruyama method with a time-step of $\Delta t=10^{-4}$, whereas the dashed black lines correspond to nodal values of deterministic simulations. The insets are over the last 10 time units. For all simulations we set $|K|=9, \sigma=0.02, \beta=1$, and $\delta=1$. We include nodal plots of the deterministic steady state values for the Square Lattice and Path graphs in (e) and (f); stochastic simulations fluctuate about these retaining the same spatial organization. In (c) at $t=50$ the central node has the deterministic value of $p_{k} \approx 0.47$, the nodes of degree 3 the value $p_{k} \approx 0.44$, and the nodes of degree 2 the value $p_{k} \approx 0.39$. In (d) at $t=50$ the boundary nodes have the deterministic value of $p_{k} \approx 0.33$, the nodes bordering the boundary have the value $p_{k} \approx 0.41$, and the central nodes of degree 2 the value $p_{k} \approx 0.4$. 


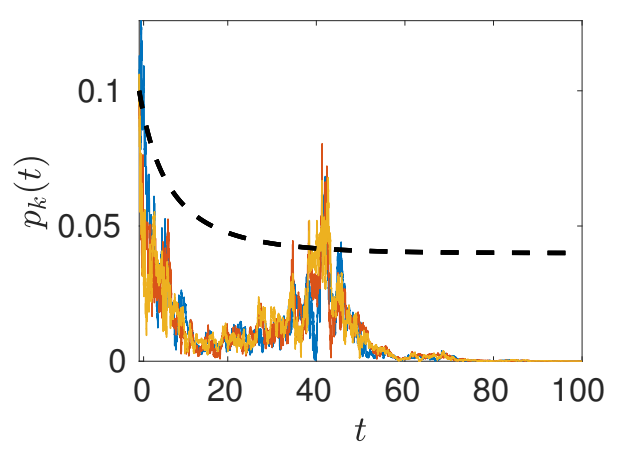

(a)

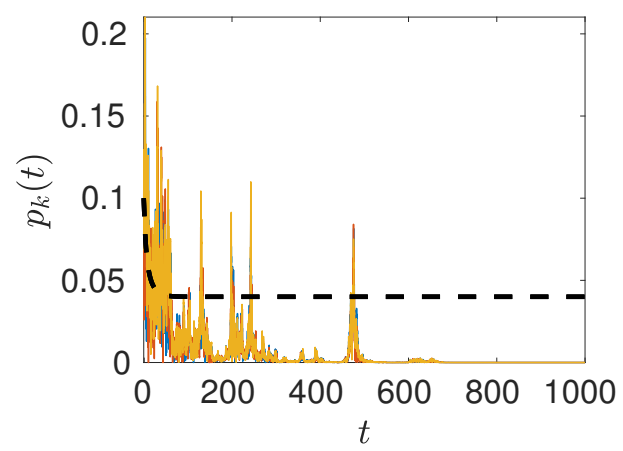

(b)

Figure 3: Time series of nodal values of the infected populations $p_{k}(t)$ on a path graph with three nodes. Solid lines correspond to stochastic simulations using Euler-Maruyama with a time-step of $\Delta t=10^{-4}$, whereas the dashed black lines correspond to nodal values of deterministic simulations. For all simulations we set $|K|=3, \sigma=0.4, \beta=1$, and $\delta=1.6$.

$n=1,2$, and we see that as $n$ increases, the path and cycle graph tend to the same asymptotic value of $\mathcal{R}_{0}$, whereas the grid and complete graphs tend toward lower values, due to the increased connectivity in these networks.

As expected, the stability results accurately capture the slope of the disease-free equilibrium in the $\beta-\delta$ plane, but we notice that the other contour lines, delineating different values of the mean contagion, have greater slopes, and vary slightly between network topologies. While the stability change given by $\lambda_{1}(B)=0$ is always a transcritical bifurcation, the value of the endemic equilibrium away from this bifurcation has a topologydependent structured illustrated by Figure 4. As we alluded to earlier, this suggests that the stochastic network model may have different behaviors which depend on on how far away from the deterministic bifurcation the model is in parameter space. We remark that the mild changes in these bifurcation plots with respect to the network topology (and on $n$ ) is primarily a result of using the contact rates given by (7), corresponding to homogeneous populations with uniform movement between nodes. More heterogeneous population or movement models will lead to greater changes in these bifurcation structures compared to our homogeneous contact model.

For the stochastic SIS model, we first need to define what we mean by "long-term" behavior, i.e. we need to establish the time scale at which we analyze the system. The scalar stochastic SIS model in Equation (12) was shown by Gray et al. (2011) to have stable stationary distributions. We expect that solutions to Equation (14) will admit stationary distributions for large enough times, but we anticipate the possibility of quasi-stationary behavior due to the complexity of our network model. Quasi-stationary distributions can be thought of as stationary states with noise-induced fluctuations, with the possibility that eventually the process may drift to an absorbing state (e.g. the disease-free equilibrium). If we eliminate the white noise by letting $\sigma \rightarrow 0$, the quasi-stationary distributions should approach stationary states of the deterministic SIS model. This can be exploited to construct asymptotic approximations to the quasi-stationary distribution in the limit of $\sigma \rightarrow 0$ (Mateus et al., 2016).
We can illustrate this idea by letting $p^{*}=1-\frac{\delta}{\beta}$ be the endemic equilibrium to Equation (2). We then expand around this equilibrium by setting $p(t)=p^{*}+\sigma \xi_{k}(t)$ for $\sigma \ll 1$ in Equation (12) to find,

$$
\sigma d \xi=\sigma \xi\left(\beta\left(1-2 p^{*}\right)-\delta\right) d t+\sigma p^{*}\left(1-p^{*}\right) d W+O\left(\sigma^{2}\right),
$$

which, upon dividing by $\sigma$ and dropping higher-order terms, is an Ornstein-Uhlenbeck process. The solution to Equation (19) approaches a stationary Gaussian distribution with mean 0 and variance $\frac{\left(p^{*}\left(1-p^{*}\right)\right)^{2}}{2\left(\beta\left(1-2 p^{*}\right)-\delta\right)}$. An analogous small-noise approximation can be made for the system (14), if the variances $\sigma_{k j}$ are taken to be of the same order, leading to a coupled system of linear stochastic differential equations. However, this system will include forcing from multiple white noise processes for each node, and will depend on the (in general heterogeneous) endemic equilibrium of Equation (6), resulting in a heterogeneous stochastic matrix system. In the interest of brevity, rather than study this asymptotic limit in a detailed manner analytically, we shall explore the behavior of solutions to the system (14) numerically.

We first consider the scalar stochastic system given by (12). We choose a sufficiently long time scale such that the deterministic solutions are very close to their equilibrium values, and consider this a transient time period $T_{1}$. We then solve (12) for a range of values of $\beta$ and $\delta$, and plot

$$
\bar{p}=\frac{1}{\left(T_{2}-T_{1}\right)} \int_{T_{1}}^{T_{2}} p_{k}(t) d t,
$$

where $T_{2}-T_{1}$ is the timescale over which we average the disease. We take $T_{1}=1000$ and $T_{2}=2000$. The value $\bar{p}$ is the long-term mean contagion. We note that for this timescale, the deterministic simulations are within numerical precision of their steady-state value. We plot the mean contagion $\bar{p}$ over $\delta$ and $\beta$ for the scalar case in Figure 5. For small values of the noise variance $\sigma^{2}$, we see essentially identical behavior to the deterministic case. This is in line with the Gaussian solution to Equation (19) for small noise, as the asymptotic behavior of the solution with small noise has a mean value identical to the deterministic process. As expected from the results in Gray et al. 

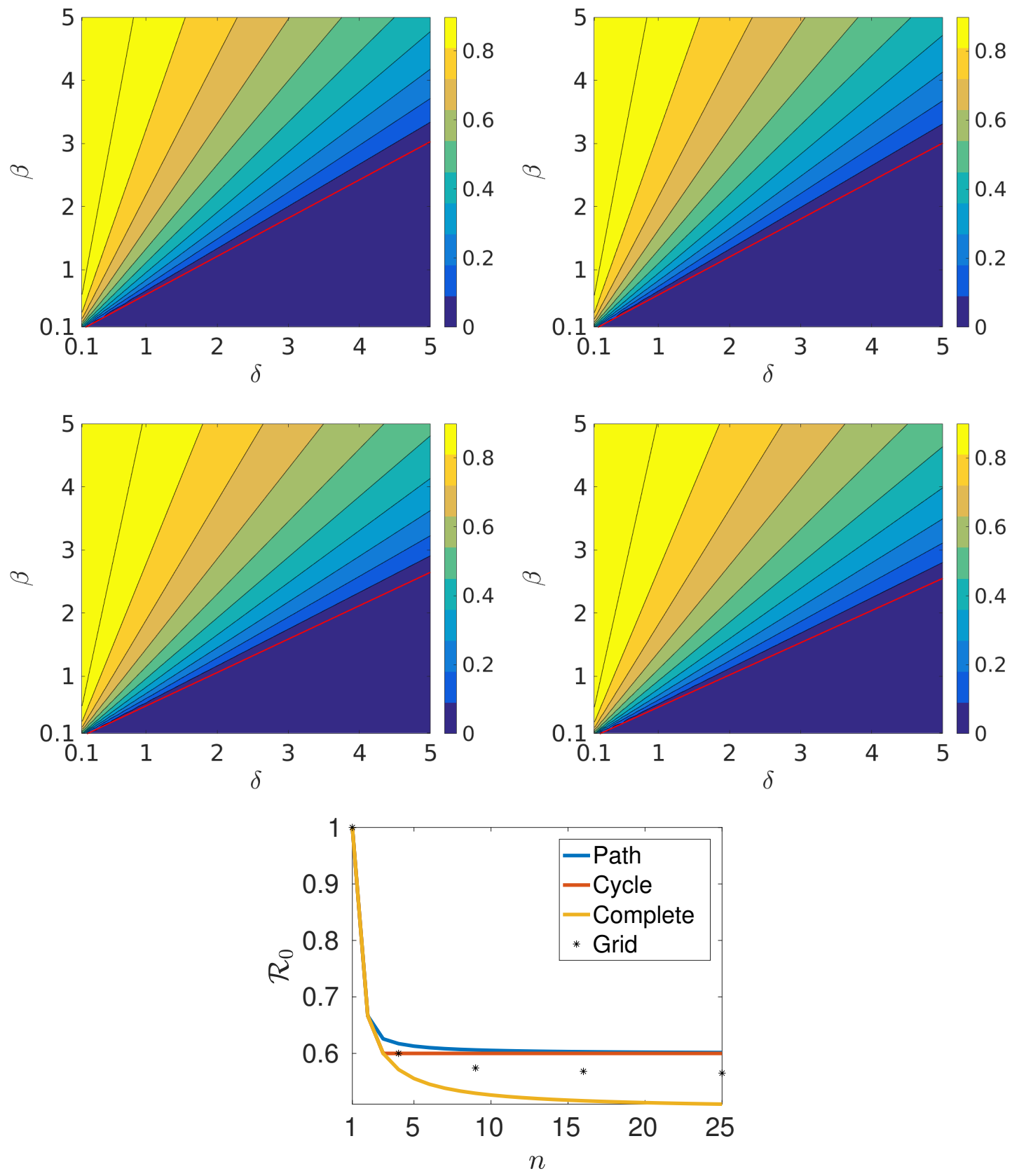

Figure 4: Bifurcation diagrams for the deterministic SIS model (given by equation (6)) on 9-node (left) or 25-node (right) networks. We consider path (top), and complete (middle) networks, as well as a characterization of the basic reproduction number $\mathcal{R}_{0}$ in the bottom plot for varying $n$. The colour scale corresponds to values of $\bar{p}$ defined in equation (18), and the red line corresponds to the deterministic value of $\lambda_{1}(B)=0$, corresponding to where the disease-free equilibrium changes stability. Topological changes correspond to shifting the slope of the stability boundary, as well as the value of the endemic equilibrium within its stability region. 

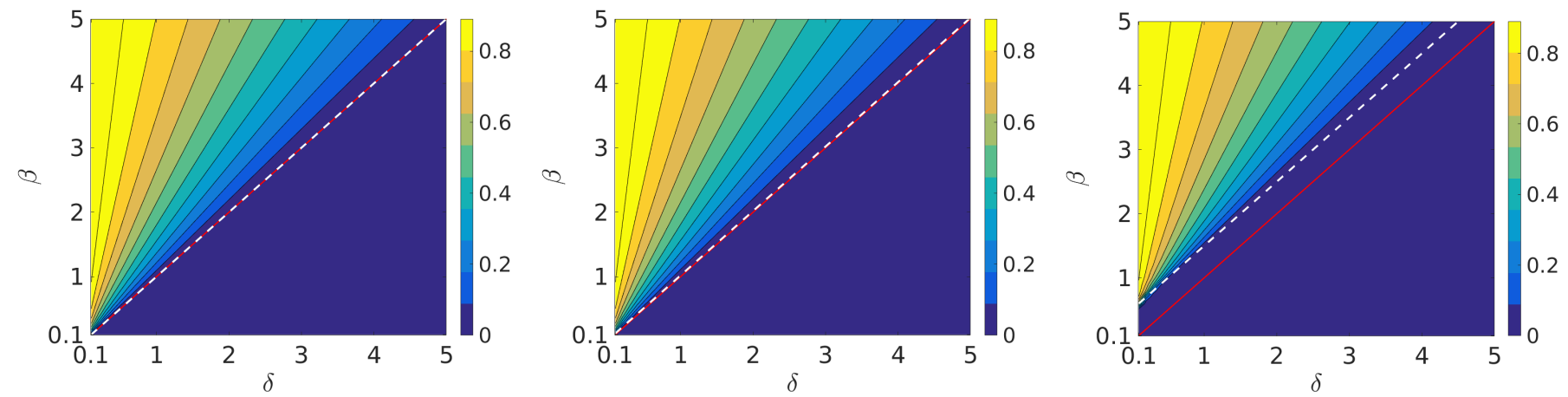

Figure 5: Bifurcation diagrams for the scalar stochastic SIS model (given by equation (12)) for values of $\sigma=0.05,0.2$, and 1 from left to right. The colour scale corresponds to values of $\bar{p}$ defined in equation (20), and the red line corresponds to the deterministic value of $\lambda_{1}(B)=0$, corresponding to where the disease-free equilibrium changes stability. The dashed white line corresponds to the stochastic stability boundary reported by Gray et al. (2011).

(2011), increasing the variance of the noise increases the likelihood of the disease dying off in finite time despite being in the endemic equilibrium stability region of the $\beta-\delta$ plane for the deterministic system, and all of our simulations confirm their noise-modified value of $\mathcal{R}_{0}$.

As we remarked before, the scalar and network stochastic cases have different noise properties. Equation (12) can always be interpreted as having multiplicative noise that approaches 0 as $p \rightarrow 0$, whereas Equations (14) can behave as if it is additively forced if $p_{k}$ is very small for some node but not small for a neighboring node. In this case, negative drift in the noise can lead to non-physical negative levels of infection, and hence spurious results. We note that this behavior only occurs for large values of the variance, namely we only observed it in the regime of $\sigma^{2}>\max \left(\beta, \beta(2 \delta)^{-1}\right)$, and suspect that the results of Gray et al. (2011) in this regime do not simply carry over. For smaller variances, the effect of the noise on the mean level of infection compared to the deterministic model is almost negligible, as can be seen in Figure 5 for the scalar case. We will discuss other noise models in Section 5 that can avoid these issues.

\section{Control Strategies}

Now that we have explored the broad behavior of stochastic SIS dynamics on networks, we shall turn our attention to several simple control strategies which may be used to contain or moderate the prevalence of the contagion. Our proposed strategies are different from those proposed in Khanafer et al. (2014), as our controls will depend on information about the current state of the disease throughout the network. One way of introducing a control into the SIS system is by effectively increasing the recovery rate (Nowzari et al., 2016). As one example, this can be achieved by treating a certain proportion of the infected population.

First consider the one-node case, described by equation (12). Let $u(t)$ be the proportion of the sick population treated at time $t$, where $0 \leq u(t) \leq 1$, and let $\alpha>0$ be the treatment effectiveness, so that $\alpha u(t)$ is the total recovery rate due to treatment. We can incorporate this control into the SIS equation (12) to obtain

$$
d p=(\beta p(1-p)-\delta p-u \alpha) d t+(\sigma p(1-p)) d W
$$

where the whole population recovers at the total rate $\delta+\alpha u(t)$. Therefore, we can view $\alpha u(t)$ as an enhancement to the natural curing or recovery rate. We also assume that we are in the endemic stability regime shown in Section 3.2 as otherwise the natural recovery rate is by itself enough to eradicate the contagion and treatment is not necessary for the long-time elimination of the disease.

For a network of $K$ nodes, we modify equation (14) so as to include control functions $u_{k}(t)$ at each node, writing

$$
\begin{aligned}
d p_{k}(t)= & \left.\left(1-p_{k}(t)\right) \sum_{j \in \mathcal{I}_{k}} \beta_{j k} p_{j}(t)-\delta_{k} p_{k}(t)-\alpha_{k} u_{k}(t) p_{k}(t)\right) d t \\
& +\left(1-p_{k}(t)\right) \sum_{j \in \mathcal{I}_{k}} \sigma_{j k} p_{j}(t) d W_{j k},
\end{aligned}
$$

with $p_{k}(0) \geq 0$ specified, where $k=1, \ldots, K$ and $\alpha_{k}$ is the treatment effectiveness or enhanced curing rate due to the treatment applied at node $k$. As before, we will assume that the curing rate is the same throughout the network, so denote $\alpha_{k}=\alpha$ for all $k$, and instead explore the impact of purely topological changes in the network structure. We will, however, assume that the control function itself may depend in some way on the topology, and hence may not be uniform throughout the network.

\subsection{Primitive bang-bang control strategies}

We first propose one particularly simple and intuitive example, a bang-bang control, where the nodal control can only be turned completely on $\left(u_{k}(t)=1\right)$, or completely off $\left(u_{k}(t)=0\right)$. We consider various approaches to determine when this control is activated based on different local and global information about the current state of the infection throughout the network. This captures realistic scenarios in combating diseases, which crucially depend on information at different scales. We also introduce a tolerance level of infection at each node $k$, denoted by $p_{k}^{*}$, that determines whether or not we administer treatment. 
This tolerance level corresponds either to an acceptable level of infection in a population, or to a level that is detectable.

\section{Case 1: Isolated Decision Making}

First, we define a control based on local information only as

$$
u_{k}^{*}(t)=\left\{\begin{array}{l}
1, \text { whenever } p_{k} \geq p_{k}^{*}, \\
0, \text { whenever } p_{k}<p_{k}^{*},
\end{array}\right.
$$

where $p_{k}^{*} \in[0,1]$ is a given tolerance level at node $k$. Whether the control at node $k$ is entirely on or off depends only on the density $p_{k}$ of the infected population at this node alone, hence the decision to implement the control is "isolated", as it does not take into account information about infection at other nodes. In the scalar deterministic setting, if the tolerance is set as $p_{k}^{*}=0$, then this control would change the basic reproduction number by a factor of $\alpha^{-1}$, which may or may not be sufficient to eradicate the disease. In the deterministic network setting, or the stochastic setting, it is not obvious a priori how the stability of the disease-free equilibrium changes with this control.

\section{Case 2: Average Neighbourhood-Based Control}

We also consider a control locally at each node that accounts for the average level of infection at all neighboring nodes. In this case we define the control functions to be

$$
u_{k}^{*}(t)=\left\{\begin{array}{l}
1, \text { whenever } \frac{1}{\left|I_{k}\right|} \sum_{j \in I_{k}} p_{j} \geq p_{k}^{*}, \\
0, \text { whenever } \frac{1}{\left|I_{k}\right|} \sum_{j \in I_{k}} p_{j}<p_{k}^{*},
\end{array}\right.
$$

for $k=1, \ldots, K$, where $\left|\mathcal{I}_{k}\right|$ is the number of elements in the index set $\mathcal{I}_{k}$, and we assume $k \in \mathcal{I}_{k}$ for all $k$. One might anticipate that this control would be more effective at eliminating epidemics given more information than the previous local control. We note that this averaging is only one possible way of determining nearby levels of infection.

\section{Case 3: Cautious Neighbourhood-Based Control}

The third case we consider is one where the control is activated as soon as the contagion reaches the tolerance level at any of the neighboring nodes. The control functions in this case are defined as

$$
u_{k}^{*}(t)= \begin{cases}1, \text { whenever } & \max _{j \in I_{k}} p_{j} \geq p_{k}^{*}, \\ 0, \text { whenever } & \max _{j \in I_{k}} p_{j}<p_{k}^{*},\end{cases}
$$

for $k=1, \ldots, K$, where $\left|\mathcal{I}_{k}\right|$ is the number of elements in the index set $\mathcal{I}_{k}$, and we assume $k \in \mathcal{I}_{k}$ for all $k$. We anticipate that this control will be activated more quickly than (23) for the same threshold infection level $p_{k}^{*}$.

\section{Case 4: Central Planning Control}

Finally, the decision when to activate the control at any node $k$ can be taken based on the prevalence of the disease across the entire network. For simplicity we consider the average infection across the network. In this case the control function is defined as

$$
u^{*}(t)=\left\{\begin{array}{l}
1, \text { whenever } \frac{1}{K} \sum_{j=1}^{K} p_{j} \geq p^{*}, \\
0, \text { whenever } \frac{1}{K} \sum_{j=1}^{K} p_{j}<p^{*},
\end{array}\right.
$$

and this control is applied at all nodes, i.e. $u_{k}^{*}(t)=u^{*}(t)$ for all $k=1, \ldots, K$.

In Figure 6, we plot the mean prevalence of the infection across the network for different graph topologies. We note that the qualitative behaviour was the same between multiple realizations. In particular, when a particular strategy was always better than another (as in Figure 6a), this was preserved among realizations. We note that this level of $\beta$ corresponds to a steady state value of the infected population much larger than $p_{k}^{*}$, and so most of the control strategies activated frequently, with little difference between the efficacy between one another. More obvious long-time differences occurred for the path and grid graphs. In all graphs we observe Case 3 being most effective, Case 2 being least effective, and the others being equally effective. Finally, we remark that most of the strategies give very comparable time series of the mean for the complete graph (when run with the same noise realization), as the information about the disease at any individual node is equivalent throughout the network, except for Case 3 which is more effective due to being activated when a single node exceeds the threshold.

In Figure 7, we consider a weaker infection with a smaller value of the treatment $\alpha$ and the same threshold. In this case, there is more variation between the treatment efficacies, as well as between the network topologies. We note that Case 3 consistently brings the mean infection substantially below the threshold $p_{k}^{*}$, Case 2 is consistently below the threshold, and Cases 1 and 4 consistently brings the mean infection very close to this threshold. We note in particular that Cases 1, 2, and 4 all additionally seem to reduce the temporal variance in the longtime behavior of the infected population, whereas Case 3 does not seem to do this as much. This result suggests an interesting trade-off between reducing the mean level of the infection or reducing the overall variability of the infection throughout the population. An additional aspect of this is that the cautious strategy, while most effective at preventing the disease, is also active much more frequently than the others.

While these results suggest global differences between these controls on different network topologies, we can also differentiate them based on how they perform nodally. In Figure 8, we consider the lattice graph with the four control cases using the same parameters as in Figure 7. We see that the isolated strategy of Case 1 leads to a uniform level of the disease approximately at the threshold throughout the network, whereas the other control strategies have more complicated effects. Case 2 (Figure $8(\mathrm{c}-\mathrm{d}))$ leads to the central node having an intermediate level of infection, the degree 3 boundary nodes having the highest level of infection, and the corner nodes having the lowest. This makes sense given the definition of Case 2 in terms of average neighboring infection populations. For similar reasons, but be- 


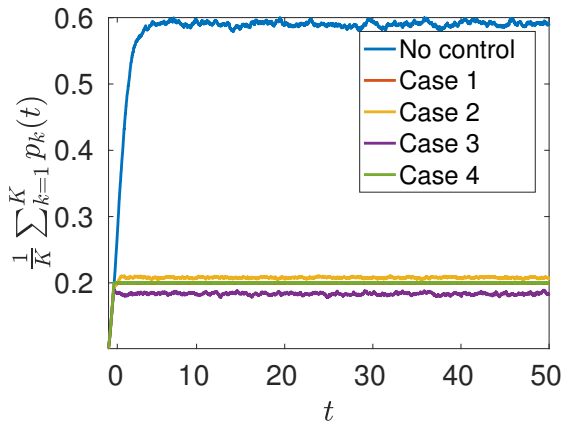

(a)

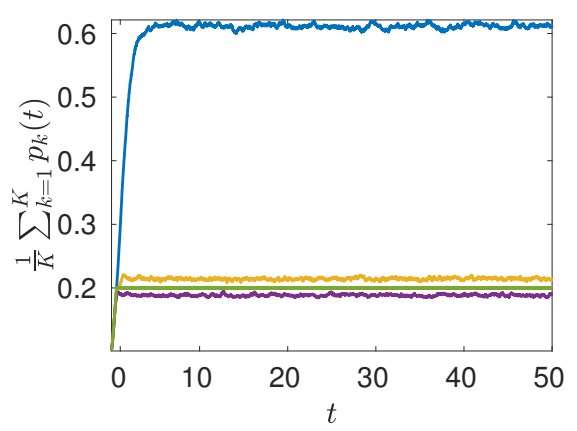

(c)

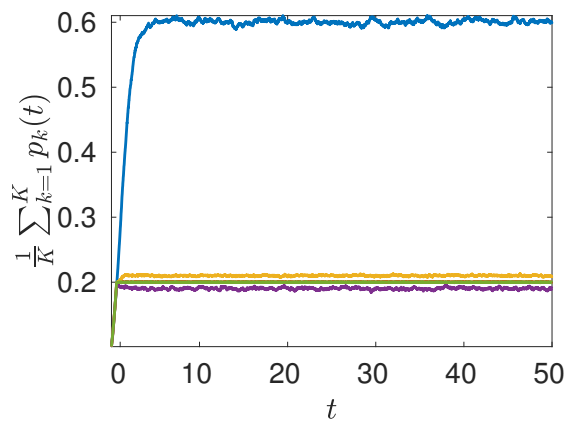

(b)

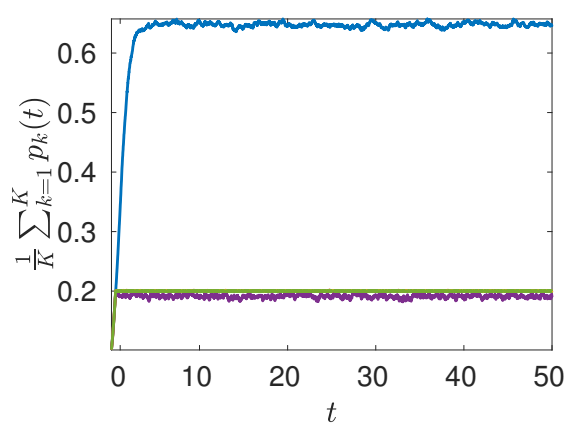

(d)

Figure 6: Solutions to system (22) with simple bang-bang control, for a path (a), cycle (b), lattice (c), and a complete graph (d) all with 9 nodes. The average of the infection across the network is shown in each case. The values of parameters are $\alpha=2, \beta=1.5, \sigma=0.05, \delta_{k}=1$, and $p_{k}^{*}=0.2$ for all $1 \leq k, m \leq K$. Note that Cases 1 and 4 are almost identical in all plots, and 1,2, and 4 are almost identical in (d).

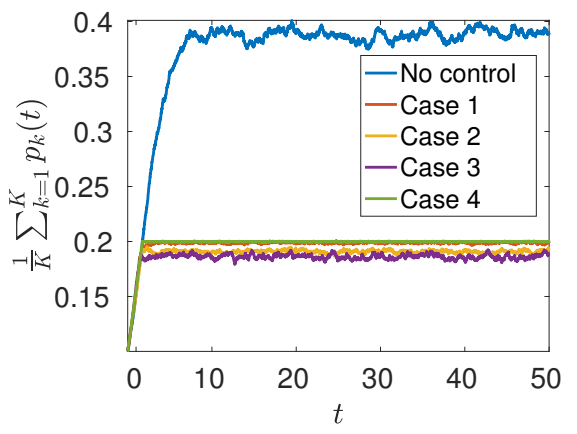

(a)

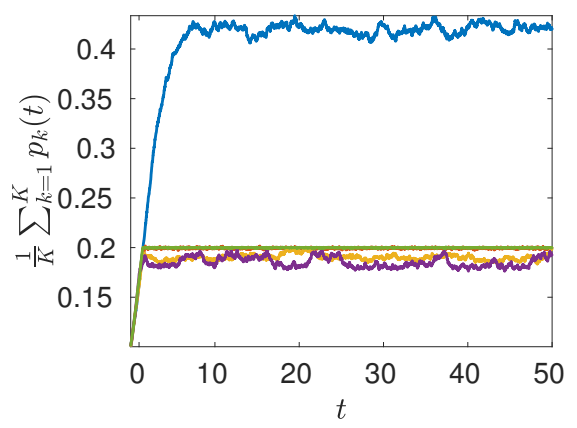

(c)

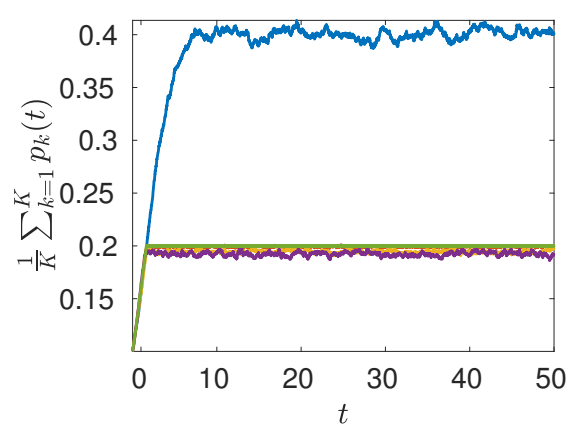

(b)

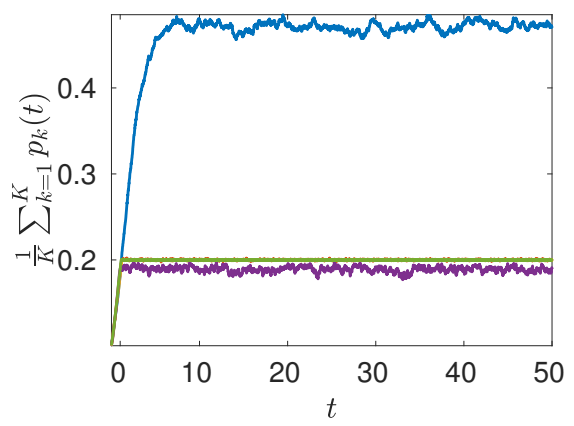

(d)

Figure 7: Solutions to system (22) with simple bang-bang control, for a path (a), cycle (b), lattice (c), and a complete graph (d) all with $|K|=9$ nodes. The average of the infection across the network is shown in each case. The values of parameters are $\alpha=1, \beta=1, \sigma=0.05, \delta_{k}=1$, and $p_{k}^{*}=0.2$ for all $1 \leq k, m \leq K$. Note that Cases 1 and 4 are almost identical in all plots, and 1,2, and 4 are almost identical in (d). 


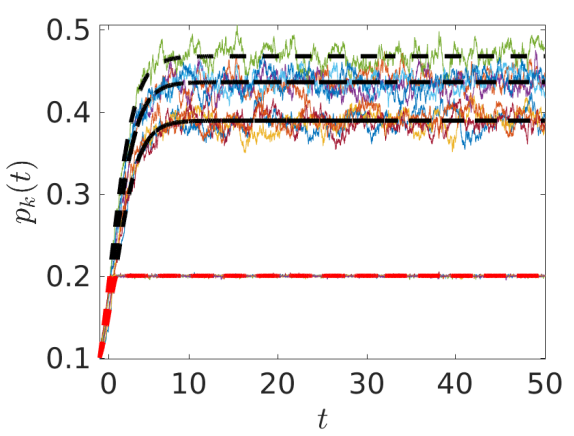

(a) Case 1

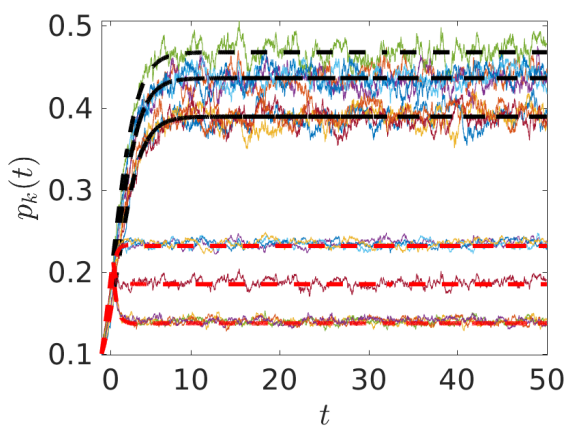

(c) Case 2

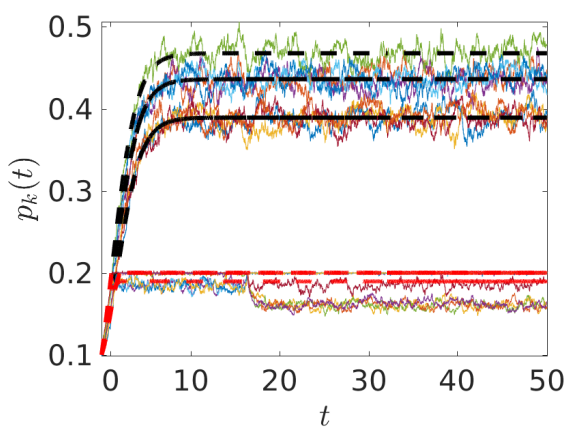

(e) Case 3

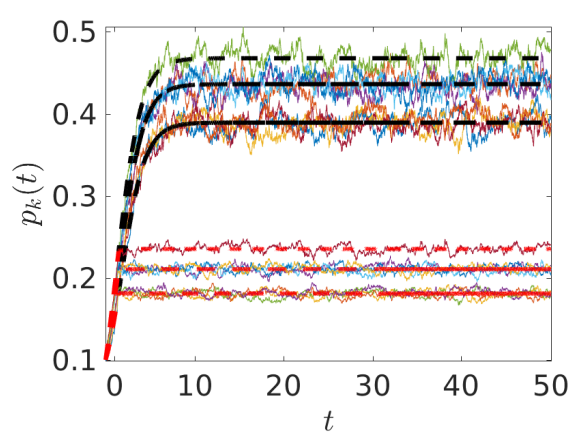

(g) Case 4

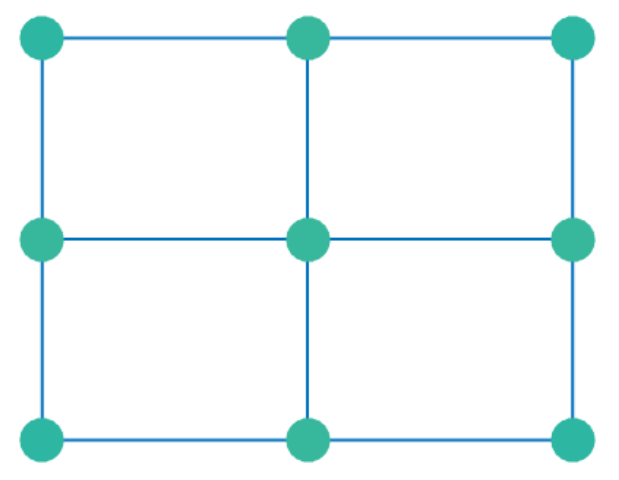

(b)

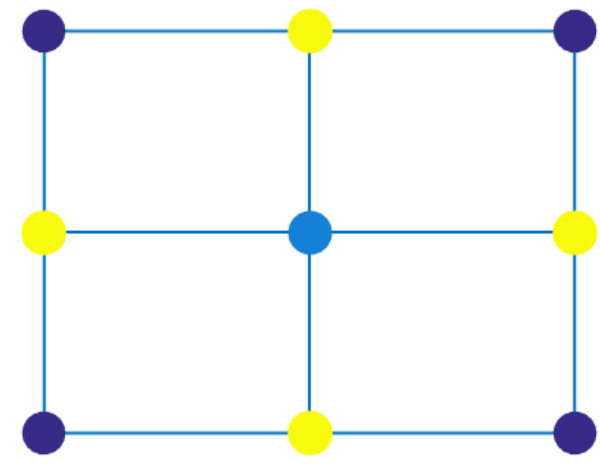

(d)

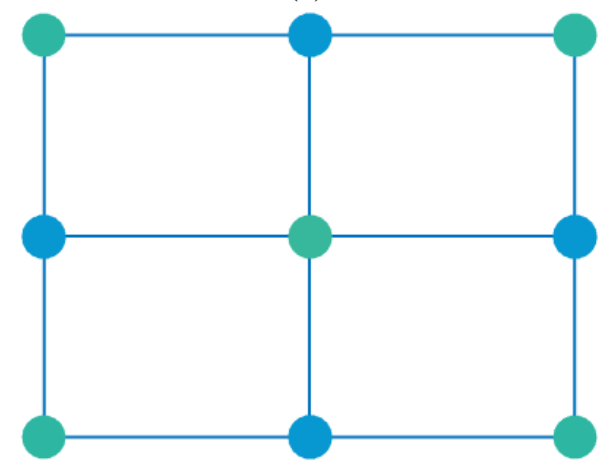

(f)

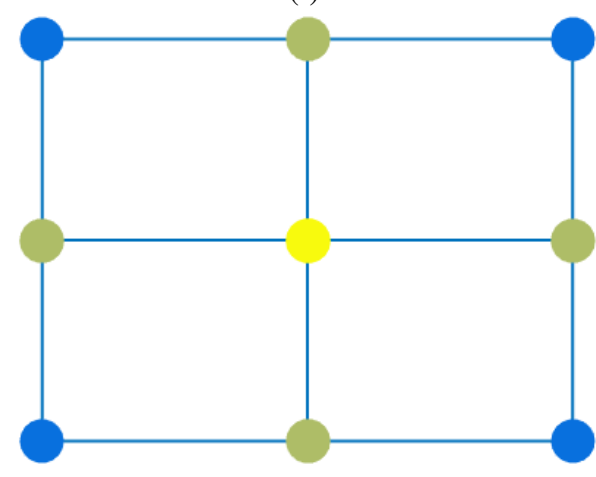

(h)

\subsection{8}

0.2

0.22

Figure 8: Solutions to system (22) with simple bang-bang control, for a lattice with 9 nodes using controls given by Cases 1-4 in each respective row. The left column are nodal timeseries with and without treatment, whereas the right at the steady state values of the deterministic simulations. The upper part of each time series is without treatment, with deterministic nodal values in dashed black lines, whereas the lower parts are with treatment at the threshold $p_{k}^{*}=0.2$ with deterministic nodal values in dashed red lines. The values of parameters are $\alpha=1, \beta=1, \sigma=0.05$, and $\delta_{k}=1$, for all $1 \leq k, m \leq K$. We note that the deterministic solutions at $t=50$ (with treatment) correspond to the nodal values shown on the right, so that the deterministic time series can be understood in terms of the long-time spatial distribution of infection throughout the lattice, and the stochastic simulations fluctuate about these values. 
cause of the overly-sensitive control strategy, in Figure 8(e-f) we see that Case 3 falls into the control consistently being activated at the degree 3 nodes, so that these have the lowest level of infection, whereas the central node and corner nodes have the highest. Finally the Case 4 simulations (Figure 8g-h) qualitatively match the no-treatment case, as the central node has the highest level of infection while the degree 2 nodes have the lowest.

\subsection{Feedback Control on Networks}

In contrast to the bang-bang controls which are either on or off, we now consider a feedback control that dynamically adjusts the treatment level based on the level of contagion present in the network. Consider

$$
\begin{aligned}
\dot{u}_{k}(t) & =F_{k}\left(\mathbf{p}(t), \mathbf{p}^{*}\right)\left(u_{k}(t)-c_{k} u_{k}(t)^{2}\right), \\
u_{k}(0) & =u_{k}^{(0)},
\end{aligned}
$$

where $u_{k}^{(0)} \in[0,1]$ is a given constant, $c_{k}>0$ is a parameter related to the cost of treatment (and hence determines the maximum allowable treatment), and $F_{k}$ is a function of the state vector $\mathbf{p}(t)=\left[p_{1}(t), \ldots, p_{K}(t)\right]^{T}$ and the threshold vector $\mathbf{p}^{*}=\left[p_{1}^{*}, \ldots, p_{K}^{*}\right]^{T}$. As was done in the case of bang-bang controls, the functions $F_{k}$ are used to determine the risk or variation of the epidemic away from some desired threshold value. Away from the threshold value, the right hand side of (27) should increase, resulting in an increase in the time derivative of the control, and hence the value of the control. The second term on the right hand side of (27) is negative for realistic (positive) costs and so serves as a limiting factor for the control. Note that this is a generic feedback control that makes intuitive sense, and alternative control laws that may be more realistic for a given situation should behave in qualitatively similar ways to (27).

For simplicity, we set $c_{k}=1$. From this we have that $0 \leq u_{k}(t) \leq 1$ for all $k=1, \ldots, K$ and $t>0$. Therefore, the effective treatment $\alpha_{k} u_{k}(t)$ will vary between 0 and $\alpha_{k}$. Further, note that if we set $u_{k}^{(0)}=0$, then $\dot{u}_{k}(0)=0$ whence $u_{k}(t)=0$ for all $t>0$. Hence we need to start off by implementing some positive level of treatment in order for the process to begin, although because of the exponential decay onto $u_{k}=0$, an arbitrarily small initial value will suffice. We set this to be $u_{k}^{(0)}=0.001$ in numerical simulations. In what follows, we shall pick values of $F_{k}$ similar to what we have considered before for bang-bang controllers, and then compare the utility of the resulting feedback controllers. These cases will be analogous to those in Section 4.1, and so we only briefly describe their interpretation within the setting of the feedback control (27).

\section{Case 1: Isolated Decision Making}

We specify the control function at node $k$ by the solution of the ODE

$$
\begin{aligned}
\dot{u}_{k}(t) & =\left(p_{k}(t)-p_{k}^{*}\right)\left(u_{k}(t)-u_{k}(t)^{2}\right), \\
u_{k}(0) & =u_{k}^{(0)}
\end{aligned}
$$

coupled with equation (22) for the evolution of the nodal infections. Equation (28) models a feedback control that adjusts to local levels of infection.

\section{Case 2: Average Neighbourhood-Based Control}

We now consider a control function at node $k$ defined by the ODE

$$
\begin{aligned}
\dot{u}_{k}(t) & =\left(\frac{1}{\left|\mathcal{I}_{k}\right|} \sum_{j \in \mathcal{I}_{k}} p_{j}-p_{k}^{*}\right)\left(u_{k}(t)-u_{k}(t)^{2}\right), \\
u_{k}(0) & =u_{k}^{(0)}
\end{aligned}
$$

coupled with equation (22) for the evolution of $p_{k}$ 's. Compare this with the equivalent type II bang-bang control (24). Note that the control function in (29) is only increasing with $t$ when the mean infection at node $k$ or its neighboring nodes reaches the tolerance level. This means that the treatment may not be activated even when the disease is very strong at one node, if its neighbors all have very low level of contagion.

\section{Case 3: Cautious Neighbourhood-Based Control}

The control function at node $k$ is defined by the solution to the ODE

$$
\begin{aligned}
\dot{u}_{k}(t) & =\left(\max _{j \in I_{k}} p_{j}(t)-p_{k}^{*}\right)\left(u_{k}(t)-u_{k}(t)^{2}\right), \\
u_{k}(0) & =u_{k}^{(0)},
\end{aligned}
$$

coupled with equation (22) for the evolution of $p_{k}$ 's. In contrast with Case 2, the control function will activate at node $k$ as soon as the contagion reaches the tolerance level at any of the neighbors of $k$ or $k$ itself. This suggests that this control should be more responsive to contagion presence and therefore more effective in reducing the endemic equilibrium.

\section{Case 4: Central Planner Control}

The control function at node $k$ is found from the ODE

$$
\begin{aligned}
\dot{u}_{k}(t) & =\left(\frac{1}{K} \sum_{j=1}^{K} p_{j}(t)-p_{k}^{*}\right)\left(u_{k}(t)-u_{k}(t)^{2}\right), \\
u_{k}(0) & =u_{k}^{(0)}
\end{aligned}
$$

coupled with equation (22) for the evolution of $p_{k}$ 's. The function $u_{k}(t)$ in this case is identical across the network, and so any topological structure of the network is ignored by this strategy.

We implement these controls at each node, and solve the resulting system of controls together with (22). We plot diagrams of the mean infection throughout the network in a high-endemic equilibrium with strong controls in Figure 9, and in a smaller endemic equilibrium with weaker controls in Figure 10, analogously to Figures 6-7. We note that, as before, Case 3 typically does the best at minimizing the mean infection throughout the network. However, unlike with the bang-bang controls shown in Figures 6-7, the variance in the time series for all controls here is noticeable. Also note that the time scale is an order of magnitude longer as the feedback control takes some time to adjust to the information about the infection, as opposed to the instantaneous response in the bang-bang setting. In particular we note that the path and grid graphs take some time to adjust to what appears to be an equilibrium distribution. We also note that generally these controls seem to be much closer to 


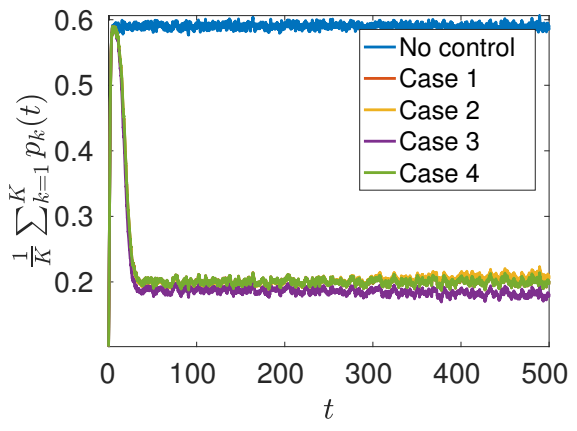

(a)

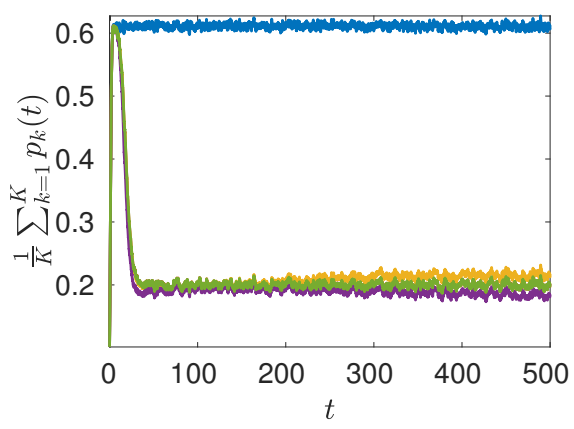

(c)

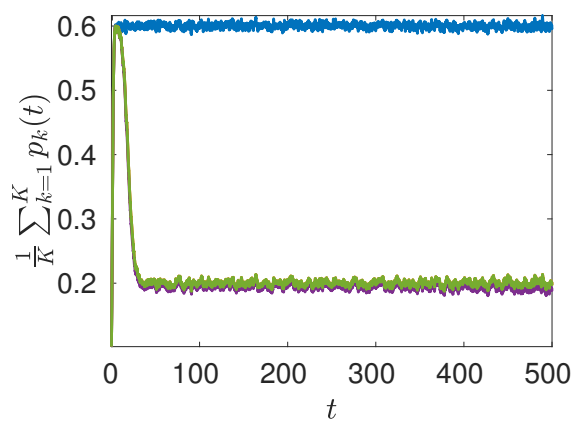

(b)

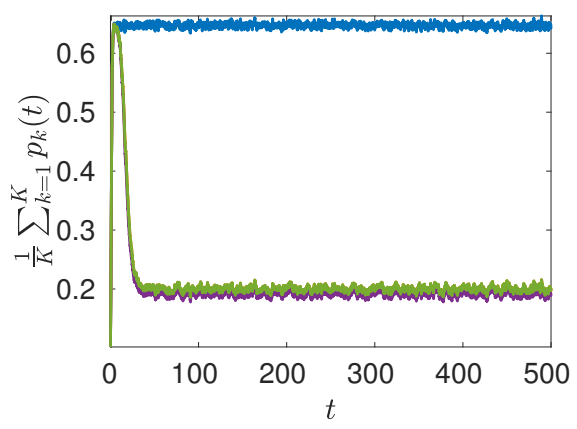

(d)

Figure 9: Solutions to equations (22) with feedback controls given by (28)-(31), for a path (a), cycle (b), lattice (c), and a complete graph (d) all with 9 nodes. The average of the infection across the network is shown in each case. The values of parameters are $\alpha=2, \beta=1.5, \sigma=0.05, \delta_{k}=1$, and $p_{k}^{*}=0.2$ for all $1 \leq k, m \leq K$. Note that Cases 1 and 4 are almost identical in all plots, and 1,2, and 4 are almost identical in (b) and (d).

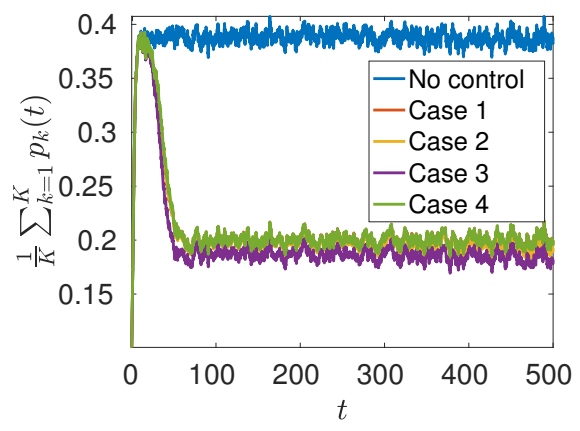

(a)

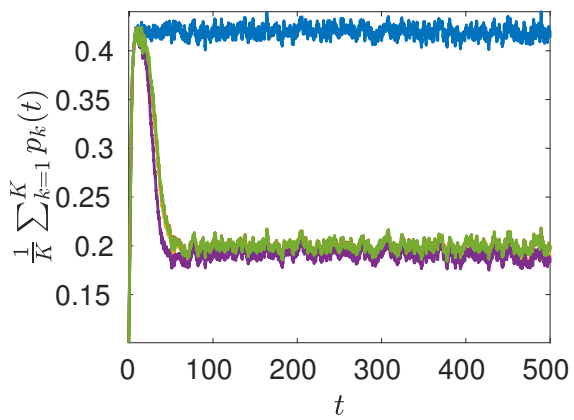

(c)

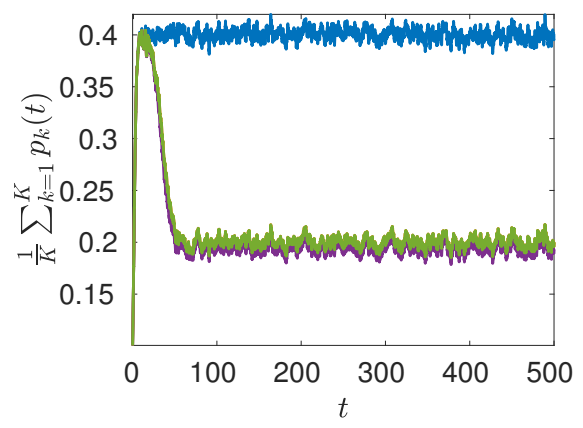

(b)

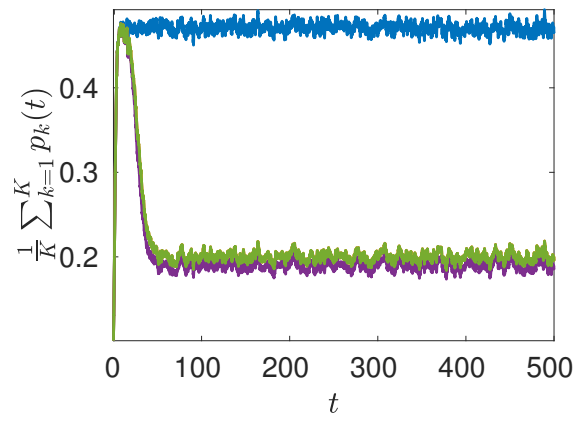

(d)

Figure 10: Solutions to equations (22) with feedback controls given by (28)-(31), for a path (a), cycle (b), lattice (c), and a complete graph (d) all with 9 nodes. The average of the infection across the network is shown in each case. The values of parameters are $\alpha=1, \beta=1, \sigma=0.05, \delta_{k}=1$, and $p_{k}^{*}=0.2$ for all $1 \leq k, m \leq K$. Note that Cases 1 and 4 are almost identical in all plots, and 1,2, and 4 are almost identical in (b)-(d). 


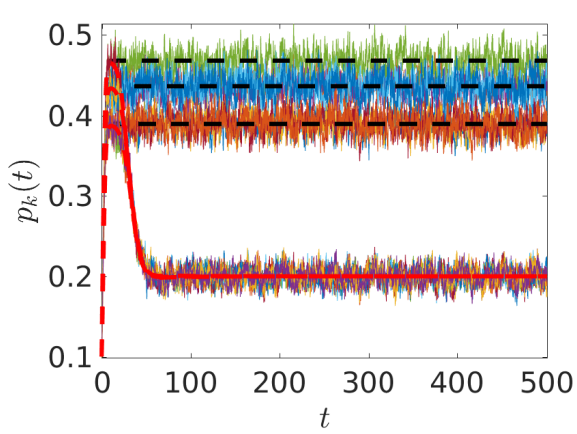

(a) Case 1

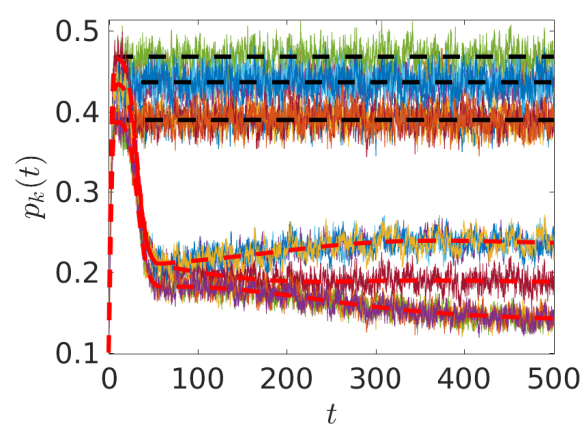

(c) Case 2

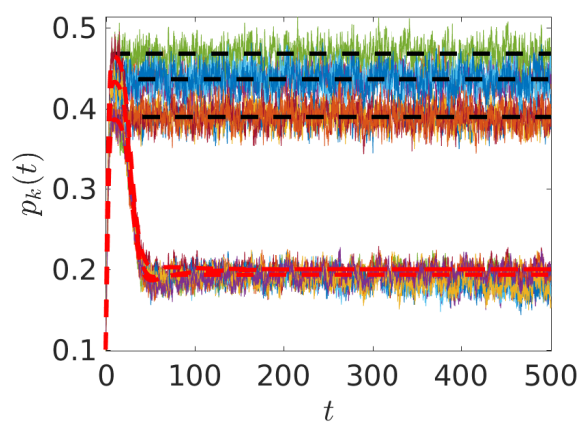

(e) Case 3

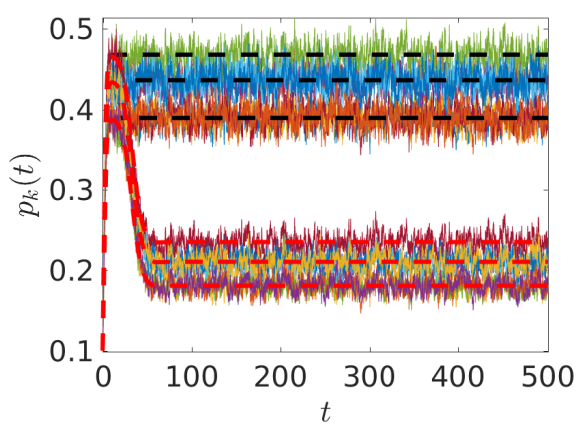

(g) Case 4

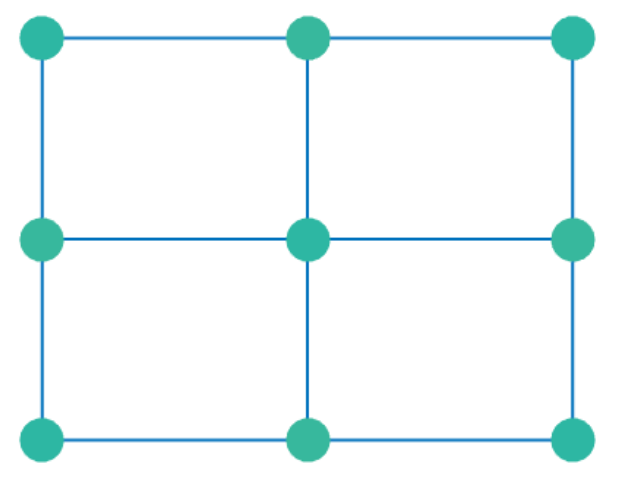

(b)

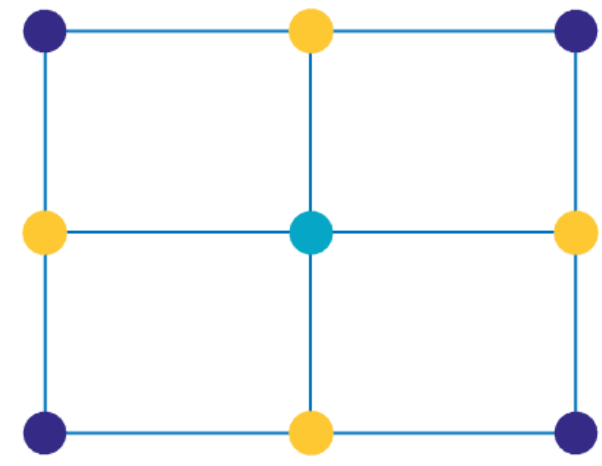

(d)

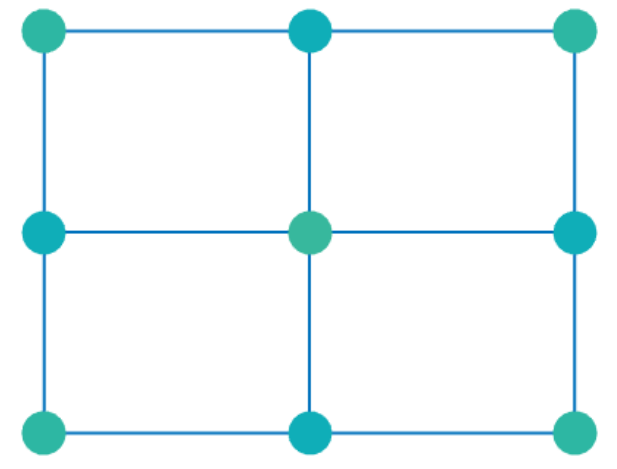

(f)

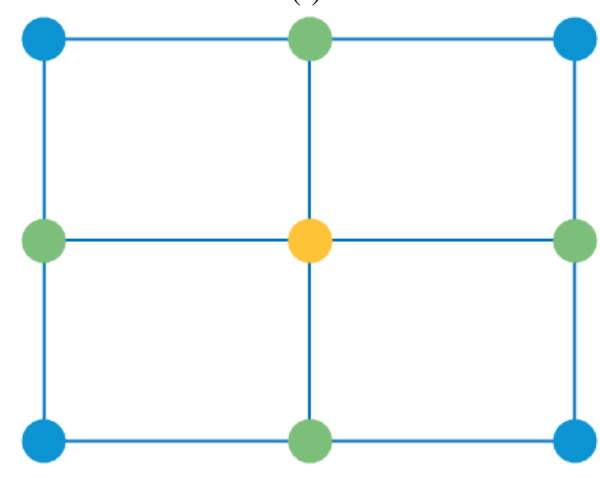

(h)

\subsection{8}

0.2

0.22

Figure 11: Solutions to system (22) with feedback control, for a lattice with 9 nodes using controls given by Cases 1-4 in each respective row. The left column are nodal timeseries with and without treatment, whereas the right at the steady state values of the deterministic simulations. The upper part of each time series is without treatment, with deterministic nodal values in dashed black lines, whereas the lower parts are with treatment at the threshold $p_{k}^{*}=0.2$ with deterministic nodal values in dashed red lines. The values of parameters are $\alpha=1, \beta=1, \sigma=0.05$, and $\delta_{k}=1$, for all $1 \leq k, m \leq K$. We note that the deterministic solutions at $t=500$ (with treatment) correspond to the nodal values shown on the right, so that the deterministic time series can be understood in terms of the long-time spatial distribution of infection throughout the lattice, and the stochastic simulations fluctuate about these values. 
one another in terms of efficacy, compared with the bang-bang controls discussed above, and hence on average it is difficult to compare them.

As before, we consider the lattice graph on 9 nodes to investigate the impact of these control strategies on the spatiotemporal behavior of the disease process throughout the network. We plot time series of stochastic and deterministic infections across a lattice graph with and without treatment in Figure 11, again in analogy with Figure 8 . We see comparable behavior as before, with the nodal level of the infection depending on both the treatment strategy and the topology of the network, sometimes leading to nodes below the disease threshold and other nodes above it. As we remarked above, this control strategy is less effective at reducing the variance in the time series, but over long periods of time it does seem to be comparable to the bang-bang control, and it may provide a more realistic model of feedback-induced treatment under different kinds of awareness of the present state of an epidemic. While qualitatively similar spatial distributions of long-time infected populations are observed in Figure 11 as in Figure 8, these emerge over a much longer timescale compared with the bang-bang controls, especially for Case 2, where the process takes longer than an order of magnitude to reach the same spatial distribution as for bang-bang controls (even in the deterministic case).

\section{Truncated Noise Model}

The stochastic model given by equation (14) is an extension of the model presented in Gray et al. (2011), and makes use of white noise to model uncertainty in the infection rate between nodes. Such parameter perturbation techniques have been studied in many epidemiological models (Chen and Li, 2009; Yang and Mao, 2013; Dalal et al., 2007; Ding et al., 2008; Tornatore et al., 2005; Lu, 2009). This technique is useful as a way of introducing temporal uncertainty in the process, and is particularly attractive because of its analytical tractability and ease of implementation. However, this method of introducing noise into the epidemic process has two disadvantages which are, as far as the authors are aware, currently unreported in the literature. The first is the problem described in Section 3.2 where for some nodal configurations, the noise term can lead to negative values of the infected population at a particular node due to the additive behavior of the noise term. This was not seen in Gray et al. (2011), as the noise in the scalar case is always multiplicative. The second problem is that white noise can induce negative infection rates.

To see this, consider a finite but small time interval, $\Delta t \ll 1$. We then can approximate the (scalar) infection rate in equation (9) as

$$
\tilde{\beta} d t=\beta d t+\sigma d W(t) \approx \beta \Delta t+\sigma \sqrt{\Delta t} \mathcal{N}(0,1),
$$

where $\mathcal{N}(0,1)$ denotes a normally distributed random variable with mean zero and unit variance. We can then compute the probability of this infection rate being negative as

$$
\operatorname{Pr}\left(\mathcal{N}(0,1)<-\frac{\beta \sqrt{\Delta t}}{\sigma}\right)=\frac{1}{\sqrt{2 \pi}} \int_{-\infty}^{-\frac{\beta \sqrt{\Delta t}}{\sigma}} e^{-\frac{x^{2}}{2}} d x \rightarrow \frac{1}{2},
$$

as $\Delta t \rightarrow 0$, suggesting that negative infection rates can be realized infinitely often. There are several alternatives to developing alternative noise models for the same kind of perturbation described by (9). Here we will give one algorithmic example, and we are presently pursuing others.

A straightforward modification of the perturbation given by (32) would be to replace the normal random variable by a truncated normal distribution. We recall the probability density function and cumulative density function of a normally distributed random variable $x$ as

$$
f(x)=\frac{1}{\sqrt{2 \pi}} e^{\frac{x^{2}}{2}}, \quad F(x)=\frac{1}{\sqrt{2 \pi}} \int_{-\infty}^{x} e^{\frac{s^{2}}{2}} d s,
$$

and say that a random variable $y \sim \mathcal{N}_{-h}^{h}(0, h)$ comes from a truncated normal distribution of standard deviation $h$ if it has the following probability density function

$$
g(y)=\left\{\begin{array}{lc}
\frac{f\left(\frac{y}{h}\right)}{F(1)-F(-1)}, & -h<y<h, \\
0, & \text { otherwise. }
\end{array}\right.
$$

It is easy to see that this random variable has zero mean and variance

$$
V(y)=h^{2}\left(1-\frac{2}{e(F(1)-F(-1))}\right) .
$$

Given this distribution, we can propose a different parameter perturbation and ensure that the contact rate is never negative for any value of the time step $\Delta t$. We replace (32) with

$$
\tilde{\beta} d t \approx \beta \Delta t+\hat{\sigma} \sqrt{\Delta t} \mathcal{N}_{-\sqrt{\Delta t}}^{\sqrt{\Delta t}}(0,1)
$$

where we scale the variance as

$$
\hat{\sigma}=\frac{\sigma}{\left(1-\frac{2}{e(F(1)-F(-1))}\right)},
$$

so that the random variable maintains the same first and second moments as the original white noise process. We now remark that this will give non-negative contact rates as long as $\hat{\sigma}<\beta$, and can easily extend to the network setting in precisely the same way as described in Section 3. In particular, we see that this truncated random variable implies that $\tilde{\beta} \in(\beta-\hat{\sigma}, \beta+\hat{\sigma})$.

While the stochastic perturbation described by (38) will not share higher moments beyond the mean and the variance with the white noise process described in the rest of this article, the effect it has on SIS dynamics is very similar. We now demonstrate this numerically using the Euler-Maruyama method mentioned in Section 3.1, fixing $\Delta t=10^{-4}$, and using the TRUNCATE function in MATLAB to generate elements from the truncated distribution. In Figure 12, we repeat the same simulations (using the same seed for the random number generator) as in Figure 2. We observe the same behavior of nodal infections fluctuating about the deterministic values, with only small differences in the frequency and amplitude of fluctuations.

Finally, we consider whether or not this form of the noise can lead to stochastic extinction, as was shown analytically in Gray et al. (2011) for the case of a white noise perturbation. Due 


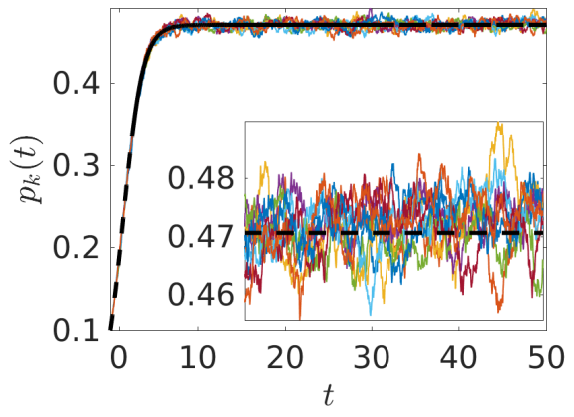

(a) Complete Graph

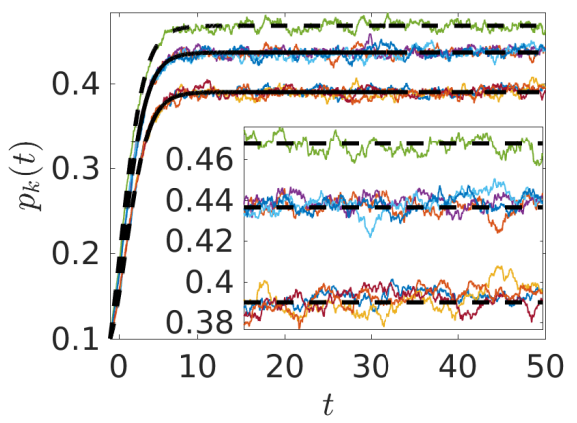

(c) Square Lattice Graph

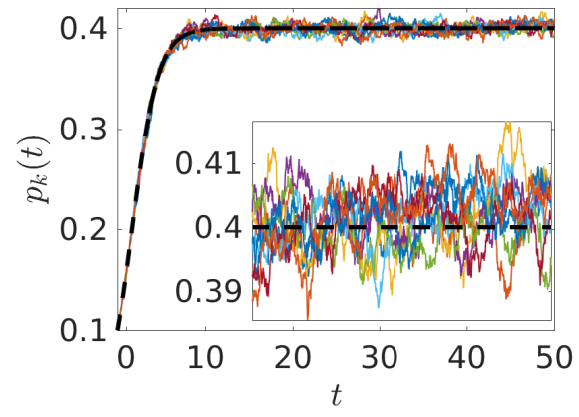

(b) Cycle Graph

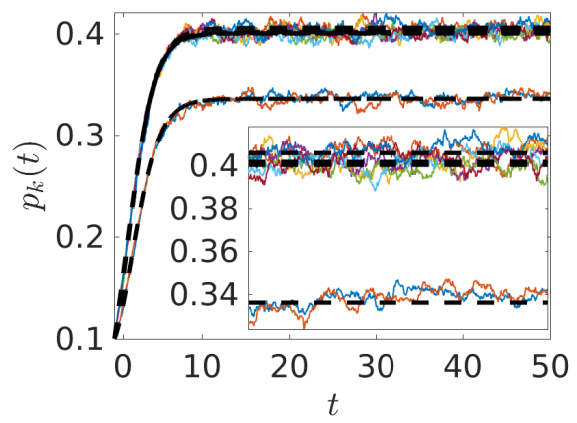

(d) Path Graph

Figure 12: Time series of nodal values of the infected populations $p_{k}(t)$ in four different graphs of the truncated white noise model. Solid lines correspond to stochastic simulations, whereas the dashed black lines correspond to nodal values of deterministic simulations. The insets are over the last 10 time units. For all simulations we set $|K|=9, \sigma=0.02, \beta=1$, and $\delta=1$. In (c) at $t=50$ the central node has the deterministic value of $p_{k} \approx 0.47$, the nodes of degree 3 the value $p_{k} \approx 0.44$, and the nodes of degree 2 the value $p_{k} \approx 0.39$. In (d) at $t=50$ the boundary nodes have the deterministic value of $p_{k} \approx 0.33$, the nodes bordering the boundary have the value $p_{k} \approx 0.41$, and the central nodes of degree 2 the value $p_{k} \approx 0.4$.

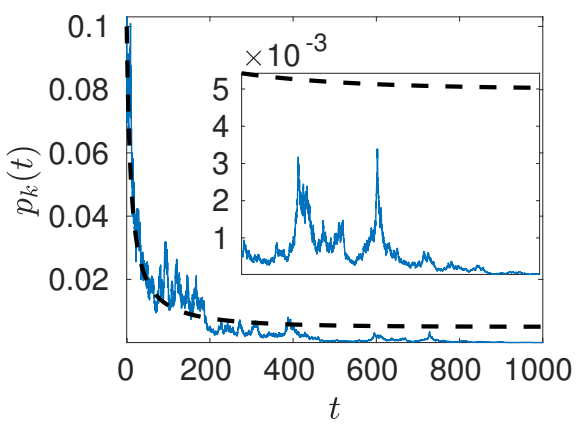

(a) Gaussian Noise

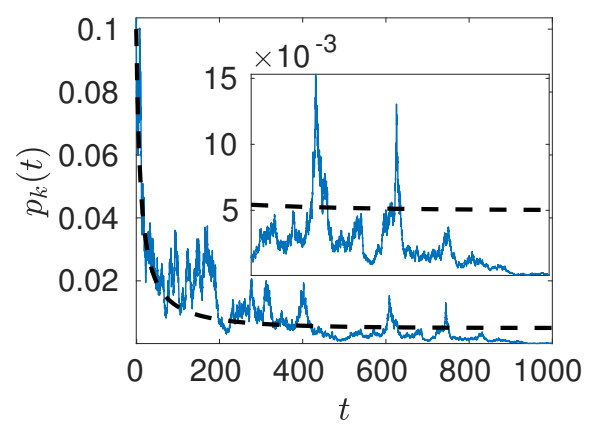

(b) Truncated Gaussian Noise

Figure 13: Time series of values of the infected populations $p$ in solutions to (12) for white noise and the truncated Gaussian process. Solid lines correspond to stochastic simulations, whereas the dashed black lines correspond to nodal values of deterministic simulations. The insets are over the last 500 time units. For all simulations we set $\sigma=0.15, \beta=1$, and $\delta=0.995$. 
to the more complicated nature of the truncated Normal distribution, especially concerning a suitable stochastic calculus, we do not pursue an analytical treatment here. Rather, we simply demonstrate stochastic extinction in the scalar case in Figure 13 via solutions with both kinds of noise. While the truncated noise process takes longer to reach a small value, repeating this simulation over $10^{4}$ units of time for $10^{2}$ simulations showed that $76 \%$ of them reached a value less than $10^{-8}$, and all of them reached a value less than $10^{-5}$ by the end of the simulation. While the value of the basic reproduction number for this truncated noise process is likely different from the value of $\mathcal{R}_{0}^{S}$ reported in Gray et al. (2011), we observe that this noise can affect the long-time behavior of an endemic stationary distribution if the volatility is large enough.

The control procedures have comparable effects using this noise model, and so we conclude that there really are not major differences in terms of the predictions given these two forms of parameter uncertainty. In particular, while white noise leads to unphysical negative contact rates infinitely often, we suspect that it does not give predictions that are substantially different from from a truncated noise process that does not have these unphysical effects. Additionally, while Brownian motion is easier to study analytically and easier to implement numerically than our proposed process, we note that there have been recent efforts in developing algorithms and other techniques to understand these kinds of probability distributions (Botev, 2017).

There are other ways of introducing stochasticity into an SIS process that not only avoid the issues described above, but also allow more accurate modelling of a stochastic contact rate. In particular, rather than use (32) (or (37)) to model the noise process, one could consider a generalized drift-diffusion model of the form,

$$
\tilde{\beta} d t=A(\tilde{\beta}, t) d t+B(\tilde{\beta}, t) d W(t),
$$

where $A$ represents an underlying deterministic drift in the contact rate, and $B$ the stochastic diffusion due to white noise. Such a general noise model allows the formulation of a wide variety of stochastic processes. In particular, mean-reverting processes, such as OrnsteinUhlenbeck or Hull-White processes (Hull and White, 1994), can provide good models of fluctuations of the contact rate around a typical value. Alternatively, a Cox-Ingersoll-Ross process (Cox et al., 2005) can be analytically shown to maintain positivity of the contact rate under certain parameter restrictions, and has been used to mimic the SIR process (Cauchemez and Ferguson, 2008). Our use of white noise in this paper is a first step toward incorporating stochasticity within deterministic metapopulation models of epidemics. We leave detailed investigation of other stochastic models as future work.

\section{Conclusions}

We have extended the stochastic SIS model in Gray et al. (2011) to spatial networks, thereby obtaining a stochastic metapopulation SIS model. We solved the stochastic model numerically and found that white noise terms introduced in the force of infection do not change the overall long-term dynamics of the system (for sufficiently small variation in the white noise), but that such white noise terms can lead to resurgence of the disease above the deterministic equilibrium, as well as to spatiotemporal heterogeneity throughout the network domain. These differences can be described as the long-time solutions tending to quasi-stationary distributions, rather than settling down to constant steady states as is seen in the deterministic analogue of the network SIS model.

We also considered approaches for controlling the spread of a stochastic SIS epidemic over a spatial network. In particular, we considered both instantaneous treatment via a simple bang-bang control, as well as a feedback control that continuously relaxed in response to information about the present state of the epidemic. In both cases we used several different kinds of information about the epidemic state of the network, corresponding to a spectrum between local and global awareness of the epidemic. We found that cautiously administering treatment if a nearby population was above a threshold level of infection tended to achieve the maximal effectiveness of the control, although this did induce some heterogeneity in the infected populations throughout the network. Depending on various other factors, such as increased risks or costs in different populations (nodes) or risks of large variances in the infection, different strategies become more appealing. Finally, we note that these are simple and generic approaches, and that a real-world solution would likely incorporate far more structure in a treatment specific to the circumstances. Hence we leave the extension of such an analysis to the metapopulation setting as future work.

Given a cost functional associated to each treatment strategy, one could compute a priori both the cost and the effectiveness of the controls presented here in order to determine the most suitable practical approach to minimizing the impact of a disease. Alternatively, optimal control strategies have been studied to address this question in stochastic epidemic models (Kovacevic, 2018). One could try to implement an optimal control using a backward-forward numerical scheme. This is numerically difficult, and perhaps a better approach is to seek low-cost, effective control functions that are not necessarily optimal but robust and intuitive to implement, as we have done here. Indeed, even for the deterministic case, full optimal control of SIS epidemics on networks is an open problem (Nowzari et al., 2016). For the stochastic analogue, design and implementation of a proper stochastic optimal controller would likely be much more difficult.

The model of stochastic SIS on networks given by Equations (14) does have some potentially non-physical behaviors for large values of the noise intensity or variance. However, we showed in Section 5 that a truncated noise model (which does not have these behaviors) gives qualitatively identical results in all of the regimes we presented. This suggests that the more analytically amenable noise driven by increments of Brownian motion is still worth considering. Still, alternative models of noise in metapopulation models could lead to better models of disease spreading within interacting populations. This would also allow for a more robust approach to exploring highvariance continuous population dynamics on networks, but we 
leave this as future work.

\section{Acknowledgments}

The authors thank both reviewers for comments which have improved the quality of the paper.

\section{References}

Allen, L., Burgin, A., 2000. Comparison of deterministic and stochastic sis and sir models in discrete time. Mathematical Biosciences 163, 1-33.

Allen, L. J. S., 2017. A primer on stochastic epidemic models: Formulation, numerical simulation, and analysis. Infectious Disease Modelling.

Arrigoni, F., Pugliese, A., 2002. Limits of a multi-patch sis epidemic model. Journal of mathematical biology 45 (5), 419-440.

Ball, F., Britton, T., House, T., Isham, V., Mollison, D., Pellis, L., Tomba, G. S., 2015. Seven challenges for metapopulation models of epidemics, including households models. Epidemics 10, 63-67.

Ball, F., Britton, T., Lyne, O., 2004. Stochastic multitype epidemics in a community of households: estimation and form of optimal vaccination schemes. Mathematical biosciences 191 (1), 19-40.

Ball, F., Sirl, D., Trapman, P., 2010. Analysis of a stochastic sir epidemic on a random network incorporating household structure. Mathematical Biosciences 224 (2), 53-73.

Berger, N., Borgs, C., Chayes, J., Saberi, A., 2005. On the spread of viruses on the internet. SODA '05 Proceedings of the sixteenth annual ACM-SIAM symposium on discrete algorithms 2005, 301-310.

Borgs, C., Chayes, J., Ganesh, A., Saberi, A., 2010. How to distribute antidote to control epidemics. Random Structures \& Algorithms 37, 204-222.

Botev, Z. I., 2017. The normal law under linear restrictions: simulation and estimation via minimax tilting. Journal of the Royal Statistical Society: Series B (Statistical Methodology) 79 (1), 125-148.

Brauer, F., 2017. Mathematical epidemiology: Past, present, and future. Infectious Disease Modelling 2, 113127.

Cauchemez, S., Ferguson, N. M., 2008. Likelihood-based estimation of continuous-time epidemic models from time-series data: application to measles transmission in london. Journal of the Royal Society Interface 5 (25), 885-897.

Chen, G., Li, T., 2009. Stability of stochastic delayed sir model. Stochastics and Dynamics 9 (02), 231-252.

Chen, L., Sun, J., 2014a. Global stability and optimal control of an sirs epidemic model on heterogeneous networks. Physica A: Statistical Mechanics and Its Applications 410, 196-204.

Chen, L., Sun, J., 2014b. Optimal vaccination and treatment of an epidemic network model. Physics Letters A 378 (41), 3028-3036.

Cox, J. C., Ingersoll Jr, J. E., Ross, S. A., 2005. A theory of the term structure of interest rates. In: Theory of Valuation. World Scientific, pp. 129-164.

Dalal, N., Greenhalgh, D., Mao, X., 2007. A stochastic model of aids and condom use. Journal of Mathematical Analysis and Applications 325, 36-53.

Diekmann, O., Heesterbeek, J. A. P., 2000. Mathematical epidemiology of infectious diseases: model building, analysis and interpretation. Vol. 5. John Wiley \& Sons.

Ding, Y., Xu, M., Hu, L., 2008. Asymptotic behavior and stability of a stochastic model for aids transmission. Applied Mathematics and Computation 204 (1), 99-108.

Eshghi, S., Khouzani, M., Sarkar, S., Venkatesh, S., 2015. Optimal patching in clustered epidemics of malware. IEEE Transactions on Networking.

Ferguson, N., Donnelly, C., Anderson, R., 2005. The foot-and-mouth epidemic in great britain: pattern of spread and impact of interventions. Science 292, 1155-1160.

Forster, G., Gilligan, C., 2007a. Optimizing the control of disease infestations at the landscape scale. Proceedings of the National Academy of Sciences 104 (12), 4984-4989.

Forster, G., Gilligan, C., 2007b. Optimizing the control of disease infestations of the landscape scale. PNAS 104, 4984-4989.

Gellner, G., McCann, K. S., Hastings, A., 2016. The duality of stability: towards a stochastic theory of species interactions. Theoretical Ecology 9 (4), $477-485$.
Gray, A., Greenhalgh, D., Hu, L., Mao, X., Pan, J., 2011. A stochastic differential equation sis epidemic model. SIAM Journal on Applied Mathematics 71, 876-902.

Grenfell, B., Harwood, J., 1997. (meta)population dynamics of infectious diseases. TREE 12, 395-399.

Grüne, L., Kloeden, P. E., 2001. Pathwise approximation of random ordinary differential equations. BIT Numerical Mathematics 41 (4), 711-721.

Heesterbeek, J. A. P., Dietz, K., 1996. The concept of ro in epidemic theory. Statistica Neerlandica 50 (1), 89-110.

Hethcote, H., Yorke, J., 1984. A simple model for gonorrhea dynamics. Lecture Notes in Biomathematics 56, 18-24.

Higham, D., 2001. An algorithmic introduction to numerical simulation of stochastic differential equations. SIAM Review 43, 525-546.

Hull, J., White, A., 1994. Numerical procedures for implementing term structure models i: Single-factor models. Journal of derivatives 2 (1), 7-16.

Keeling, M., Eames, K., 2005. Networks and epidemic models. Journal of the Royal Society Interface 2 (4), 295-307.

Keeling, M., Ross, J., 2008. On methods for studying stochastic disease dynamics. Journal of the Royal Society Interface 5, 171181

Keeling, M. J., Bjørnstad, O. N., Grenfell, B. T., 2004. Metapopulation dynamics of infectious diseases. In: Ecology, genetics and evolution of metapopulations. Elsevier, pp. 415-445.

Keeling, M. J., Danon, L., Vernon, M. C., House, T. A., 2010. Individual identity and movement networks for disease metapopulations. Proceedings of the National Academy of Sciences 107 (19), 8866-8870.

Keeling, M. J., Rohani, P., 2002. Estimating spatial coupling in epidemiological systems: a mechanistic approach. Ecology Letters 5 (1), 20-29.

Kermack, W., McKendrick, A., 1927. A contribution to the mathematical theory of epidemics. Proceedings of the Royal Society of London A: mathematical, physical and engineering sciences 115, 700-721.

Khanafer, A., Basar, T., Gharesifard, B., 2014. Stability properties of infected networks with low curing rates. In: American Control Conference (ACC), 2014. IEEE, pp. 3579-3584.

Khanafer, A., Basar, W., 2014. An optimal control problem over infected networks. Proceedings of the International Conference of Control, Dynamics Systmes and Robotics 2014, 125.

Kim, J., Radhakrishnan, S., Jang, J., 2006. Cost optimization in sis model of worm infection. ETRI Journal 28 (5), 692-695.

Kiss, I. Z., Berthouze, L., Taylor, T. J., Simon, P. L., 2012. Modelling approaches for simple dynamic networks and applications to disease transmission models. In: Proc. R. Soc. A. Vol. 468. The Royal Society, pp. 13321355 .

Kloeden, P. E., Neuenkirch, A., 2007. The pathwise convergence of approximation schemes for stochastic differential equations. LMS journal of Computation and Mathematics 10, 235-253.

Kovacevic, R. M., 2018. Stochastic contagion models without immunity: their long term behaviour and the optimal level of treatment. Central European Journal of Operations Research, 1-27.

Lajmanovich, A., Yorke, J. A., 1976. A deterministic model for gonorrhea in a nonhomogeneous population. Mathematical Biosciences 28 (3), 221-236.

Lu, Q., 2009. Stability of sirs system with random perturbations. Physica A: Statistical Mechanics and Its Applications 388 (18), 3677-3686.

Martcheva, M., 2015. Introduction to Mathematical Epidemiology. Vol. 61. Springer.

Mateus, L., Ghaffari, P., Skwara, U., Rocha, F., Aguiar, M., Masoero, D., Stollenwerk, N., 2016. Semiclassical approximations of stochastic epidemiological processes towards parameter estimation using as prime example the sis system with import. Ecological Complexity 27, 63-73.

McCormack, R. K., Allen, L. J. S., 2006. Stochastic sis and sir multihost epidemic models. Hindawi Publishing Company, pp. 775-786.

Newman, M., 2002. Spread of epidemic disease on networks. Physical Review E 66 (1), 016128.

Newman, M., 2010. Networks: an introduction. OUP Oxford.

Nowzari, C., Preciado, V., Pappas, G., 2016. Analysis and control of epidemics: A survey of spreading processes on complex networks. IEEE Control Systems 36 (1), 26-46.

Øksendal, B., 2003. Stochastic differential equations. Springer.

Pastor-Satorras, R., Castellano, C., Van Mieghem, P., Vespignani, A., 2015. Epidemic processes in complex networks. Reviews of Modern Physics 87 (3), 925.

Pellis, L., Ball, F., Bansal, S., Eames, K., House, T., Isham, V., Trapman, P., 
2015. Eight challenges for network epidemic models. Epidemics 10, 58-62.

Riley, S., Eames, K., Isham, V., Mollison, D., Trapman, P., 2015. Five challenges for spatial epidemic models. Epidemics 10, 68-71.

Sanders, J., Noble, B., Van Gorder, R. A., Riggs, C., 2012. Mobility matrix evolution for an sis epidemic patch model. Physica A: Statistical Mechanics and its Applications 391 (24), 6256-6267.

Shaw, L., Schwartz, I., 2010. Enhanced vaccine control of epidemics in adaptive networks. Physical Review E 81 (4), 046120.

Tomovski, I., Kocarev, L., 2012. Simple algorithm for virus spreading control on complex networks. IEEE Transactions on Circuits and Systems I: Regular Papers 59 (4), 763-771.

Tornatore, E., Buccellato, S. M., Vetro, P., 2005. Stability of a stochastic sir system. Physica A: Statistical Mechanics and its Applications 354, 111-126.

Van Mieghem, P., 2011. The n-intertwined sis epidemic network model. Computing 93 (2-4), 147-169.

Van Mieghem, P., Omic, J., 2013. In-homogeneous virus spread in networks. arXiv preprint arXiv: 1306.2588

Van Mieghem, P., Omic, J., Kooij, R., 2009. Virus spread in networks. IEEE/ACM Transactions on Networking 17 (1), 1-14.

Yang, Q., Mao, X., 2013. Extinction and recurrence of multi-group seir epidemic models with stochastic perturbations. Nonlinear Analysis: Real World Applications 14 (3), 1434-1456. 